\title{
Supersymmetric holography on AdS3
}

\section{Journal Article}

\section{Author(s):}

Candu, Constantin; Gaberdiel, Matthias R.

Publication date:

2013-09

Permanent link:

https://doi.org/10.3929/ethz-b-000073956

\section{Rights / license:}

Creative Commons Attribution 4.0 International

\section{Originally published in:}

Journal of High Energy Physics 2013(9), https://doi.org/10.1007/JHEP09(2013)071 


\section{Supersymmetric holography on $\mathrm{AdS}_{3}$}

\section{Constantin Candu and Matthias R. Gaberdiel}

Institut für Theoretische Physik, ETH Zürich, CH-8093 Zürich, Switzerland

E-mail: ccandu@gmail.com, gaberdiel@itp.phys.ethz.ch

ABSTRACT: The proposed duality between Vasiliev's supersymmetric higher spin theory on $\mathrm{AdS}_{3}$ and the 't Hooft limit of the $2 \mathrm{~d} \mathcal{N}=2$ superconformal Kazama-Suzuki models is analysed in detail. In particular, we show that the partition functions of the two theories agree in the large $N$ limit.

Keywords: AdS-CFT Correspondence, Conformal and W Symmetry, Extended Supersymmetry

ARXIV EPRINT: 1203.1939 


\section{Contents}

1 Introduction 1

2 Non-supersymmetric duality 3

2.1 The higher spin gravity theory 3

2.2 The coset point of view 4

2.3 The character identity 6

$\begin{array}{lll}2.4 & \text { Free field realisation } & 10\end{array}$

$\begin{array}{lll}3 & \text { Supersymmetric duality } & \mathbf{1 3}\end{array}$

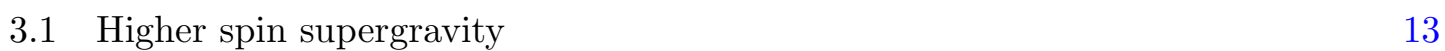

$\begin{array}{ll}3.2 \text { The superconformal coset } & 15\end{array}$

$\begin{array}{lll}3.3 & \text { The duality } & 19\end{array}$

$\begin{array}{ll}\text { 3.3.1 The higher spin partition function } & 19\end{array}$

3.3.2 The superconformal partition function 20

$\begin{array}{lll}3.4 & \text { Free field realisation } & 23\end{array}$

4 Conclusions $\quad 25$

$\begin{array}{ll}\text { A Identities for } \mathfrak{g l}(\infty \mid \infty)_{+} & \mathbf{2 5}\end{array}$

\section{Introduction}

Theories containing an infinite number of (massless) higher spin currents are an interesting class of theories that lie in complexity somewhere between field and string theories. The first non-trivial examples where constructed about twenty-five years ago by Fradkin \& Vasiliev [1, 2]. Recently, these theories have gained prominence in the context of the AdS/CFT correspondence since they are believed to be dual to free conformal theories [3-6]. This offers the hope of finding simplified versions of the AdS/CFT duality. It may also open the way towards a proof of the AdS/CFT correspondence, at least in a specific regime; for first attempts in this direction see [7-10].

About ten years ago it was conjectured by Klebanov \& Polyakov [11] (see also [12] for a subsequent refinement) that a specific higher spin theory on $\mathrm{AdS}_{4}$ [13] (see for example [14-17] for reviews) is dual to the large $N$ limit of the $\mathrm{O}(N)$ vector model in 3 dimensions; actually, there are four different versions of this duality, depending on whether one considers the free or interacting $\mathrm{O}(N)$ theory, and whether it is based on fermions or bosons. During the last two years, highly non-trivial evidence in favour of this conjecture has been found. In particular, Giombi \& Yin managed to calculate some 3-point functions 
of the higher spin theory on $\mathrm{AdS}_{4}$, and showed that they reproduce precisely those of the dual $\mathrm{O}(N)$ vector model in the large $N$ limit [18-20]. For the interacting theory, the higher spin symmetry gets broken at finite $N$ [21], but the symmetry may still play a useful role in determining the correlators of the theory.

The argument of [21] only applies to $3 \mathrm{~d}$ conformal field theories, whereas in 2 dimensions it is known that interacting higher spin theories (even with a finite number of degrees of freedom) exist, for example, the $W_{N}$ minimal models. A little while ago, it was shown that the asymptotic symmetry algebra of higher spin theories on $\operatorname{AdS}_{3}[22,23]$ lead to classical $W_{N}$ or $W_{\infty}$ symmetry algebras [24-27], and a 1-loop calculation [28] suggested that the corresponding statement would also be true for the quantum theory. A concrete proposal was then made in [29], relating the large $N$ 't Hooft like limit of the $W_{N}$ level $k$ minimal models to a family of bosonic higher spin theories on $\mathrm{AdS}_{3}$. By now quite some evidence has been found in favour of this proposal [30-36]. The proposal is the natural analogue of the Klebanov-Polyakov duality since, for vanishing 't Hooft coupling, the CFT can be described as the singlet sector of a free theory [37]. There have also been interesting results concerning the construction of black holes for these higher spin theories, as well as their dual CFT interpretation [38-41].

The proposal of [29] was generalised to the case where instead of the $\mathfrak{s u}(N)$ based $W$ algebras, one considers the $\mathfrak{s o}(2 N)$ series [42, 43]. More recently, a $\mathcal{N}=2$ supersymmetric generalisation has been proposed [44], relating a family of Kazama-Suzuki models [45, 46] to the supersymmetric higher spin theory of $[22,23]$. It is the aim of this paper to give substantial evidence in favour of this proposal; in particular, we shall give the supersymmetric generalisation of the calculation of [30], establishing the agreement between the 1-loop partition function of the supersymmetric higher spin theory on $\mathrm{AdS}_{3}$, and the partition function of the dual $\mathcal{N}=2$ superconformal field theories in the large $N$ limit. While the general strategy is similar to what was done in [30], there is one new ingredient in our analysis: unlike the bosonic $W_{N}$ case, explicit formulae for the coset characters of the Kazama-Suzuki models do not appear to be readily available. In this paper we therefore calculate them from first principles in the 't Hooft limit. The basic idea is to relate them to the branching functions of the free $(\lambda=0)$ theory which can be determined by combinatorial methods. We first apply this approach to the bosonic case, thereby reproducing the results of [30], and then use it for the supersymmetric Kazama-Suzuki models.

The paper is organised as follows. In section 2 we review the bosonic duality; in particular, we explain in detail how the partition function of the minimal models can be calculated from first principles in the 't Hooft limit, using a combinatorial approach (see section 2.4). In section 3 we then apply the same techniques to the 't Hooft limit of the Kazama-Suzuki models. Finally, section 4 contains our conclusions and an outlook towards future directions. We have relegated some of the technical arguments for the calculation of the branching and restriction rules for $\mathfrak{g l}(\infty \mid \infty)_{+}$(that play a role for the supersymmetric analysis) to an appendix. 


\section{Non-supersymmetric duality}

In this section we briefly review the non-supersymmetric duality and rederive the relation between the partition functions. Our strategy follows essentially [30], but we employ a somewhat different technique for extracting explicit formulae for the coset characters in the 't Hooft limit. This method will generalise directly to the supersymmetric case.

\subsection{The higher spin gravity theory}

Let us begin by fixing some conventions. We parametrise the Euclidean $\mathrm{AdS}_{3}$ space with coordinates $(r, z) \in \mathbb{R} \times \mathbb{C}$, for which the metric takes the form

$$
\mathrm{d} s^{2}=\frac{\mathrm{d} r^{2}+\mathrm{d} z \mathrm{~d} \bar{z}}{r^{2}}
$$

and the boundary is located at $r=0$. In thermal AdS the points $(r, z+\mathbb{Z}+\mathbb{Z} \tau)$ are identified, and the boundary becomes a torus with modular parameter $q=e^{2 \pi i \tau}$. We shall first consider the non-supersymmetric truncation of Vasiliev's higher spin theory $[22,23]$ on $\mathrm{AdS}_{3}$. This theory has massless gauge fields of spin $s=2,3, \ldots$ Assuming periodic boundary conditions around the thermal circle, a real gauge field with integer spin $s$ contributes to the 1-loop partition function the factor

$$
Z_{\text {gauge }}^{s}=\prod_{n=s}^{\infty} \frac{1}{\left|1-q^{n}\right|^{2}} .
$$

This was first calculated for the graviton $(s=2)$ in [47]; the general result was then derived in [28] using the techniques of [48].

In addition to these massless higher spin gauge fields, the theory that is proposed to be dual to the 't Hooft limit of the minimal model also contains two massive complex scalar fields [29]. A complex scalar field $\phi$ of mass squared $M^{2}$ contributes to the partition function the factor [47]

$$
Z_{\text {scalar }}^{\Delta}=\prod_{m, n=0}^{\infty} \frac{1}{\left(1-q^{h+m} \bar{q}^{h+n}\right)^{2}},
$$

provided its asymptotic behaviour near the AdS boundary is fixed to be $\phi(r, z, \bar{z}) \sim$ $a(z, \bar{z}) r^{\Delta}$. Here $\Delta=2 h$ is related to the mass squared $M^{2}$ by the familiar relation

$$
(\Delta-1)^{2}=1+M^{2} .
$$

In the duality of [29] $M^{2}=-1+\lambda^{2}$, and then there are two solutions for $\Delta$,

$$
\Delta_{ \pm}^{B}(\lambda)=1 \pm \lambda
$$

According to the proposal of [29], one complex scalar is quantised with $(+)$ boundary conditions, the other with $(-)$ boundary conditions. Then the total 1-loop partition function of the higher spin theory equals

$$
Z_{1 \text {-loop }}^{\lambda}=Z_{\text {scalar }}^{\Delta_{+}^{B}(\lambda)} \times Z_{\text {scalar }}^{\Delta_{-}^{B}(\lambda)} \times \prod_{s=2}^{\infty} Z_{\text {gauge }}^{s} .
$$

It was conjectured in [29] that this higher spin theory is dual to a specific limit of minimal model CFTs that we shall now review. 


\subsection{The coset point of view}

Consider the coset conformal field theory

$$
\frac{\mathfrak{s u}(N)_{k} \oplus \mathfrak{s u}(N)_{1}}{\mathfrak{s u}(N)_{k+1}}
$$

for integer level $k$. Its chiral algebra is the $W_{N}$ algebra of central charge

$$
c=(N-1)\left(1-\frac{N(N+1)}{(N+k)(N+k+1)}\right),
$$

which we denote as $W_{N, k}$. The primaries of the coset CFT (2.7) can be described in the usual manner [49, 50].

In order to do so explicitly, let us introduce the following notation. We denote by $Y$ the set of all Young diagrams, and by $Y_{N} \subset Y$ the subset of diagrams with less than $N$ rows; as is well known the elements of $Y_{N}$ label the representations of $\mathfrak{s u}(N)$. The representations of the affine algebra $\mathfrak{s u}(N)_{k}$ at level $k$ are then described by the diagrams $Y_{N, k} \subset Y_{N}$ that have in addition less or equal to $k$ columns.

For $\Lambda \in Y_{N, k}$ and $\omega \in Y_{N, 1}$ consider the decomposition of the tensor product in terms of representations $\Xi \in Y_{N, k+1}$ of $\mathfrak{s u}(N)_{k+1}$

$$
\Lambda \otimes \omega=\bigoplus_{\Xi}(\Lambda ; \Xi) \otimes \Xi
$$

where $\mathfrak{s u}(N)_{k+1}$ is diagonally embedded into $\mathfrak{s u}(N)_{k} \oplus \mathfrak{s u}(N)_{1}$, and $(\Lambda ; \Xi)$ denotes the corresponding multiplicity space. It is clear that only those $\Xi \in Y_{N, k+1}$ can appear in (2.9) for which the weights satisfy

$$
\Lambda+\omega-\Xi \in Q_{N},
$$

where $Q_{N}$ is the root lattice of $\mathfrak{s u}(N)$. For $\mathfrak{s u}(N)$, this equation determines $\omega$ uniquely in terms of $\Lambda$ and $\Xi$. The multiplicity spaces $(\Lambda ; \Xi)$ can thus be labelled by just $\Lambda$ and $\Xi$, and they carry, by construction, an action of the coset CFT (2.7). The coset CFT is rational and all its highest weight representations can be obtained in this manner; however, not all pairs $(\Lambda ; \Xi)$ define inequivalent coset representations, since there are field identifications [51-53].

Let us denote the characters of the $\mathfrak{s u}(N)_{k}$ and $W_{N, k}$ representations as

$$
\operatorname{ch}_{\Lambda}^{N, k}\left(q, e^{H}\right)=\operatorname{tr}_{\Lambda} q^{L_{0}} e^{H}, \quad b_{\Lambda ; \Xi}^{N, k}(q)=\operatorname{tr}_{(\Lambda ; \Xi)} q^{L_{0}} .
$$

Here $L_{0}$ is the zero mode of the energy momentum tensor in the corresponding chiral algebra, while $H$ is an element of the Cartan subalgebra of $\mathfrak{s u}(N)$. As a consequence of (2.9), we have the basic relation

$$
\operatorname{ch}_{\Lambda}^{N, k}\left(q, e^{H}\right) \operatorname{ch}_{\omega}^{N, 1}\left(q, e^{H}\right)=\sum_{\Xi} b_{\Lambda ; \Xi}^{N, k}(q) \operatorname{ch}_{\Xi}^{N, k+1}\left(q, e^{H}\right),
$$

which we will use below in order to compute the characters of the coset theory.

The simplest coset CFT is the usual charge-conjugation theory, whose Hilbert space consists of

$$
\mathcal{H}^{N, k}=\bigoplus_{[\Lambda ; \Xi]}(\Lambda ; \Xi) \otimes \overline{(\Lambda ; \Xi)}
$$




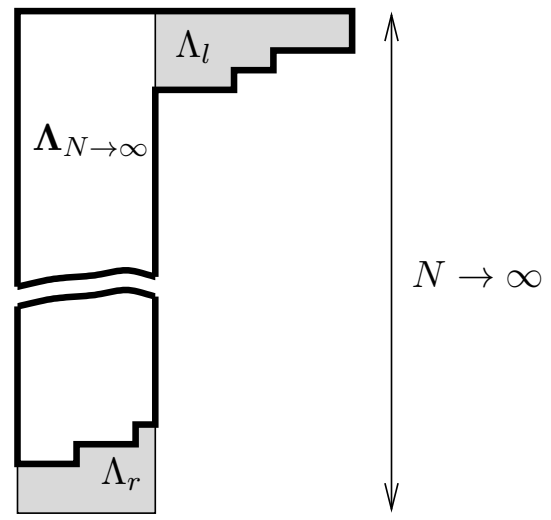

Figure 1. Young diagrams that are finite only in the horizontal direction, and that have a single infinite vertical step label $\mathfrak{s u}(N)$ representations generated by the tensor product of finitely many fundamental and dual representations in the limit $N \rightarrow \infty$.

where the two tensor factors are representations of the left- and right-moving coset CFT, respectively, and the sum is taken over isomorphism classes $[\Lambda ; \Xi]$ of representations identified by the field identification. The corresponding modular invariant torus partition function is then

$$
Z^{N, k}(q)=\left|q^{-\frac{c}{24}}\right|^{2} \sum_{[\Lambda ; \Xi]}\left|b_{\Lambda ; \Xi}^{N, k}(q)\right|^{2}
$$

It was proposed in [29] that the non-supersymmetric higher spin theory of Vasiliev is dual to the 't Hooft like large $N, k$ limit of the coset CFTs (2.13),

$$
N, k \rightarrow \infty \quad \text { with } \quad \frac{N}{N+k}=\lambda \text { held fixed. }
$$

A strong argument in favour of this proposal is the fact that the partition function (2.6) can be reproduced from the dual CFT in this limit. The way this happens is however quite intricate, since the naive limit of the partition function (2.14) diverges. In order to make sense of the limit theory it was proposed in [29] to restrict the Hilbert space (2.13) to those coset representations for which both $\Lambda$ and $\Xi$ are contained in the $N \rightarrow \infty$ limit of finite tensor powers of the fundamental representation of $\mathfrak{s u}(N)$ and its dual. Intuitively this means that in the limit both $\Lambda$ and $\Xi$ are described by a pair of Young diagrams, see figure 1 .

In order to explain this more precisely, it is convenient to think of these labels in terms of $\mathfrak{u}(N)$ representations. Recall that irreducible (tensorial) representations of $\mathfrak{u}(N)$ are labelled by pairs of Young diagrams $\boldsymbol{\Lambda}=\left(\Lambda_{l}, \Lambda_{r}\right)$ of the form represented in figure 2 . Every $\mathfrak{u}(N)$ tensor $\boldsymbol{\Lambda}$ defines an $\mathfrak{s u}(N)$ tensor labelled by a single Young diagram in $Y_{N}$, which we denote by $\boldsymbol{\Lambda}_{N}=\left(\Lambda_{l}, \Lambda_{r}\right)_{N}$. Since we can move the position where we separate $\boldsymbol{\Lambda}_{N}$ into $\Lambda_{l}$ and $\Lambda_{r}$, there are many $\mathfrak{u}(N)$ tensors $\boldsymbol{\Lambda}$ that restrict to the same $\mathfrak{s u}(N)$ tensor $\boldsymbol{\Lambda}_{N}$, but differ in their $\mathfrak{u}(1)$ charge $|\boldsymbol{\Lambda}|_{-}=\left|\Lambda_{r}\right|-\left|\Lambda_{l}\right|$, where $\left|\Lambda_{l, r}\right|$ is the number of boxes in the corresponding diagrams.

The representations we are interested in are those where we keep $\Lambda_{l}$ and $\Lambda_{r}$ fixed as we take the $N \rightarrow \infty$ limit; the resulting $\mathfrak{s u}(N)$ representation becomes then an infinite Young 


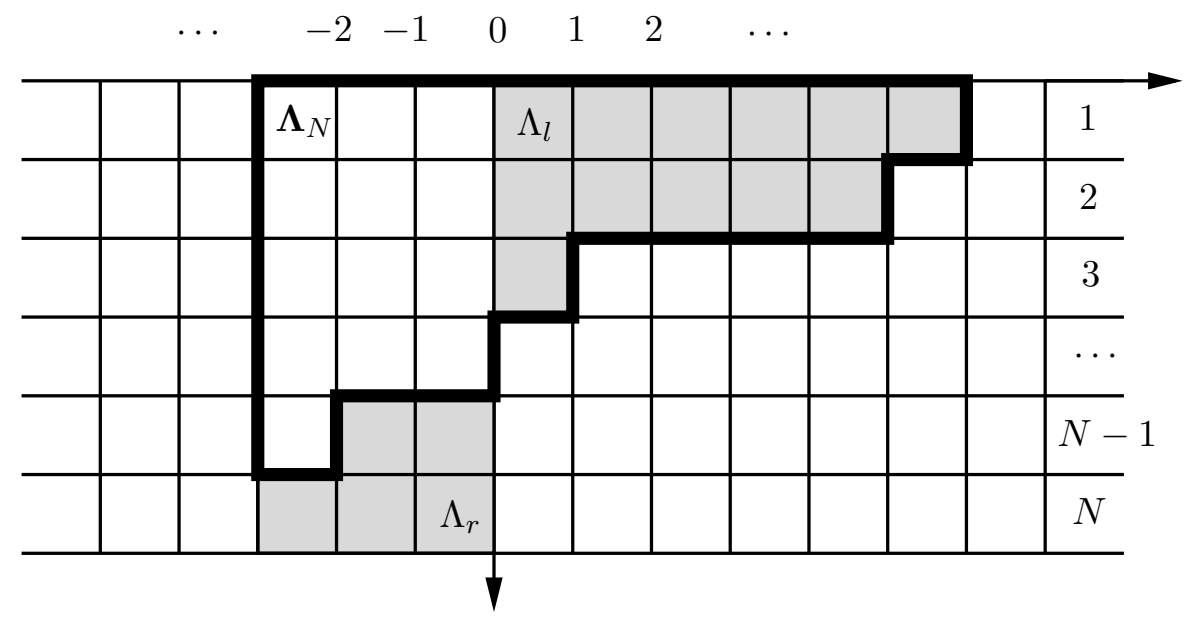

Figure 2. A $\mathfrak{u}(N)$ representation is labelled by a pair of (finite) Young diagrams $\boldsymbol{\Lambda}=\left(\Lambda_{l}, \Lambda_{r}\right)$ such that the sum of their rows is at most $N$. The corresponding $\mathfrak{s u}(N)$ dominant weight is represented by the Young diagram with a bold contour, denoted by $\boldsymbol{\Lambda}_{N}$.

diagram depicted in figure 1. As can be seen from this figure, one can unambiguously recover back from this infinite Young diagram the original pair of finite Young diagrams $\boldsymbol{\Lambda}$. From now on we shall identify the set of these infinite Young diagrams $\boldsymbol{\Lambda}_{\infty}=\left(\Lambda_{l}, \Lambda_{r}\right)_{\infty}$ with the set $\mathbf{Y}=Y \times Y$ of pairs of Young diagrams, and denote its elements by bold upper case Greek letters (such as $\boldsymbol{\Lambda}$ ).

Returning to the limit theory of (2.13), it was proposed in [29] that the Hilbert space reproducing the partition function of the dual $\mathrm{AdS}_{3}$ theory in the limit (2.15) is

$$
\mathcal{H}^{\lambda}=\bigoplus_{\boldsymbol{\Lambda}, \boldsymbol{\Xi} \in \mathbf{Y}}(\boldsymbol{\Lambda} ; \boldsymbol{\Xi}) \otimes \overline{(\boldsymbol{\Lambda} ; \boldsymbol{\Xi})}
$$

where the two pairs of Young diagrams $\boldsymbol{\Lambda}=\left(\Lambda_{l}, \Lambda_{r}\right)$ and $\boldsymbol{\Xi}=\left(\Xi_{l}, \Xi_{r}\right)$ label representations

$$
(\boldsymbol{\Lambda} ; \boldsymbol{\Xi})=\lim _{N, k \rightarrow \infty}\left(\boldsymbol{\Lambda}_{N} ; \boldsymbol{\Xi}_{N}\right)
$$

of the limit algebra $W_{\infty}[\lambda][26]$.

The second complication comes from the fact that the representations (2.17) generically become reducible in the limit (2.15), at least if both $\boldsymbol{\Lambda}$ and $\boldsymbol{\Xi}$ are non-trivial. Another way of saying this is that new null states appear in the limit that have to be removed in order to calculate the partition function. Subtracting out these contributions, it was argued in [30] that the resulting partition function of (2.16) reproduces precisely (2.6).

We would now like to give a modified version of the proof and then generalise it to the supersymmetric case.

\subsection{The character identity}

In order to make contact with eq. (2.16), the first step of the argument is to rewrite the bulk partition function (2.6) as a sum over finite Young diagrams. Let us begin by introducing 
a little bit of notation. Let $\mathfrak{g l}(\infty)_{+}$be the Lie algebra of infinite-dimensional matrices for which only finitely many diagonals adjacent to the main diagonal are non-zero. These matrices have a natural action on the infinite-dimensional vector space $\mathbb{C}^{\mathbb{N}_{0}}=\oplus_{j=0}^{\infty} \mathbb{C} e_{j}$, where $e_{j}$ denotes a basis and $\mathbb{N}_{0}$ are the non-negative integers. This is the 'fundamental' representation of $\mathfrak{g l}(\infty)_{+}$, and the representations we are interested in are those that are contained in finite tensor powers of this fundamental representation. All of these tensor products are completely decomposable, and hence we can label these representations by finite Young diagrams $\Lambda$.

We shall need to calculate the character of the representation $\Lambda$. In general, a character can be evaluated on an arbitrary element of the Cartan subgroup of the associated group GL $(\infty)_{+}$. The Cartan subgroup consists of the diagonal matrices, and the Cartan subalgebra of $\mathfrak{g l}(\infty)_{+}$can thus also be identified with the diagonal matrices; a natural basis for the Cartan subalgebra is $H_{i}=E_{i i}$, where $i \in \mathbb{N}_{0}$ and $E_{i i}$ is the matrix with a single non-zero entry in position $(i, i)$. The dual to the Cartan subalgebra is the weight space, and it is generated by the weights $\epsilon_{i}$ with

$$
\epsilon_{i}\left(H_{j}\right)=\delta_{i j} .
$$

With these preparations we can now describe the character of the representation $\Lambda$. A basis for the vector space associated to $\Lambda$ is labelled by the different Young tableaux $\operatorname{Tab}_{\Lambda}$ of shape $\Lambda$. Here a Young tableaux of shape $\Lambda$ is a Young diagram $\Lambda$ together with a filling of the boxes of $\Lambda$ by elements from $N_{0}$, where, as usual, within each row the entries of the boxes do not decrease, while within each column they increase. The weight $\operatorname{wt}(T)$ of the basis element associated to $T \in \mathrm{Tab}_{\Lambda}$ is the sum of the associated weights $\epsilon_{j}$, where $j$ runs over the entries of the boxes in the tableau $T$. Then the character of $\Lambda$ equals

$$
\operatorname{ch}_{\Lambda}\left(e^{H}\right)=\sum_{T \in \operatorname{Tab}_{\Lambda}} e^{\operatorname{wt}(T)(H)}
$$

where $H$ is an arbitrary element of the Cartan subalgebra of of $\mathfrak{g l}(\infty)_{+}$. In the following we shall mainly evaluate this character on the specific elements

$$
U(h)=\prod_{j \in \mathbb{N}_{\mathbb{0}}} e^{2 \pi i \tau(h+j) H_{j}}, \quad q=e^{2 \pi i \tau}
$$

of the Cartan subgroup of $\mathrm{GL}(\infty)_{+}$with matrix elements

$$
U(h)_{j j}=q^{h+j},
$$

where $h$ is some real number, and $q$ has modulus less than one. In this case the character (2.19) takes the form

$$
\operatorname{ch}_{\Lambda}(U(h))=\sum_{T \in \operatorname{Tab}_{\Lambda}} \prod_{j \in T} q^{h+j} .
$$

With the help of the matrix $U(h)$, we can now write the partition function of a real scalar field on thermal $\mathrm{AdS}_{3}$ as the determinant

$$
\prod_{m, n=0}^{\infty} \frac{1}{1-q^{h+m} \bar{q}^{h+n}}=\frac{1}{\operatorname{det}\left(1-U(h) \otimes U(h)^{*}\right)},
$$


where $U(h)^{*}$ is obtained from $U(h)$ upon replacing $q \mapsto \bar{q}$. This can be decomposed into $\mathfrak{g l}(\infty)_{+}$characters by performing the same manipulations as in $[30]^{1}$

$$
\frac{1}{\operatorname{det}\left(1-U(h) \otimes U(h)^{*}\right)}=\sum_{\Lambda} \operatorname{ch}_{\Lambda}(U(h)) \overline{\operatorname{ch}_{\Lambda}(U(h))} .
$$

The partition function (2.6) can thus be written as

$$
Z_{1 \text {-loop }}^{\lambda}=Z_{\text {gauge }} \sum_{\Lambda_{l}, \Lambda_{r}, \Xi_{l}, \Xi_{r}}\left|\operatorname{ch}_{\Lambda_{l}}\left(U_{+}\right) \operatorname{ch}_{\Lambda_{r}}\left(U_{+}\right) \operatorname{ch}_{\Xi_{l}}\left(U_{-}\right) \operatorname{ch}_{\Xi_{r}}\left(U_{-}\right)\right|^{2},
$$

where $\Lambda_{l}, \Lambda_{r}, \Xi_{l}, \Xi_{r}$ are finite Young diagrams, and we have defined

$$
Z_{\text {gauge }}=\prod_{s=2}^{\infty} Z_{\text {gauge }}^{s}
$$

with $Z_{\text {gauge }}^{s}$ given in (2.2). Finally, $U_{ \pm} \equiv U\left(h_{ \pm}\right)$, with $h_{ \pm}=\frac{1}{2}(1 \pm \lambda)$.

The next step is to reproduce eq. (2.25) from the coset point of view. To this end we need to evaluate the coset characters up to powers of $q^{k}$ or $q^{N}$, which become irrelevant in the 't Hooft limit. We want to determine the coset characters from (2.12), and thus we first need to understand the characters of affine representations associated to $\Lambda \in Y_{N, k}$. It follows from the Kac-Weyl formula (see e.g. [30, 55]) that we have

$$
\operatorname{ch}_{\Lambda}^{N, k}\left(q, e^{H}\right)=\frac{q^{h_{\Lambda}^{N, k}}\left[\operatorname{ch}_{\Lambda}^{N}\left(e^{H}\right)+\mathcal{O}\left(q^{k-\Lambda_{1}+1}\right)\right]}{\prod_{n=1}^{\infty}\left[\left(1-q^{n}\right)^{N-1} \prod_{\alpha \in \Delta_{N}}\left(1-q^{n} e^{\alpha(H)}\right)\right]},
$$

where $\Delta_{N}$ denotes the roots of $\mathfrak{s u}(N)$, and $\Lambda_{1}$ is the length of the first row of $\Lambda$. Here we have used that, for large $k$, only the elements of the finite Weyl group contribute to the dominant term, thus making the finite $\mathfrak{s u}(N) \operatorname{character} \operatorname{ch}_{\Lambda}^{N}$ appear. The conformal dimension of the affine primary field labelled by $\Lambda$ equals

$$
h_{\Lambda}^{N, k}=\frac{\operatorname{Cas}(\Lambda)}{2(k+N)}=\frac{N|\Lambda|}{2(k+N)}-\frac{|\Lambda|^{2}}{2 N(k+N)}+\sum_{\varepsilon \in \Lambda} \frac{\operatorname{col}(\varepsilon)-\operatorname{row}(\varepsilon)}{k+N},
$$

where $|\Lambda|$ is the number of boxes in $\Lambda$, and the sum in the last term runs over the individual boxes of the Young diagram $\Lambda$, where $\operatorname{row}(\varepsilon)$ and $\operatorname{col}(\varepsilon)$ is the row and column number of the box $\varepsilon \in \Lambda$, respectively.

Using (2.27) for the different characters in (2.12) we thus obtain

$$
\operatorname{ch}_{\Lambda}^{N}\left(e^{H}\right) \operatorname{ch}_{\omega}^{N, 1}\left(q, e^{H}\right)=\sum_{\Xi \in Y_{N}} a_{\Lambda ; \Xi}^{N}(q) \operatorname{ch}_{\Xi}^{N}\left(e^{H}\right)
$$

where we have defined the $k$ independent function $a_{\Lambda ; \Xi}^{N}(q)$; it is related to the coset character in the $k \rightarrow \infty$ limit as

$$
b_{\Lambda ; \Xi}^{N, k}(q)=q^{h_{\Lambda}^{N, k}-h_{\Xi}^{N, k+1}}\left[a_{\Lambda ; \Xi}^{N}(q)+\mathcal{O}\left(q^{k-\Lambda_{1}+1}\right)+\mathcal{O}\left(q^{k-\Xi_{1}+2}\right)\right] .
$$

\footnotetext{
${ }^{1}$ This expansion formally defines the Schur functions (with an infinite number of variables) in the theory of symmetric functions [54]. Their explicit expression as a sum over monomials labelled by Young tableaux of fixed shape and, thus the identification with $\mathfrak{g l}(\infty)+$ characters, is then an a posteriori fact.
} 
Next we observe that for $\Lambda=0$ eq. (2.29) simplifies to

$$
\operatorname{ch}_{\omega}^{N, 1}\left(q, e^{H}\right)=\sum_{\Xi \in Y_{N}} a_{0 ; \Xi}^{N}(q) \operatorname{ch}_{\Xi}^{N}\left(e^{H}\right),
$$

i.e. $a_{0 ; \Xi}^{N}(q)$ is the branching function of an $\mathfrak{s u}(N)_{1}$ affine representation into representations of the zero mode algebra $\mathfrak{s u}(N)$. In order to describe the general case, recall that the decomposition of $\mathfrak{s u}(N)$ tensor products implies that

$$
\operatorname{ch}_{\Lambda_{1}}^{N} \operatorname{ch}_{\Lambda_{2}}^{N}=\sum_{\Lambda_{3} \in Y_{N}} c_{\Lambda_{1} \Lambda_{2}}^{(N) \Lambda_{3}} \operatorname{ch}_{\Lambda_{3}}^{N}
$$

where $c_{\Lambda_{1} \Lambda_{2}}^{(N) \Lambda_{3}}$ are the Clebsch-Gordan coefficients. Multiplying (2.31) by $\operatorname{ch}_{\Lambda}^{N}$ we thus conclude that

$$
a_{\Lambda ; \Xi}^{N}(q)=\sum_{\Pi \in Y_{N}} c_{\Lambda \Pi}^{(N) \Xi} a_{0 ; \Pi}^{N}(q)=\sum_{\Pi \in Y_{N}} c_{\Lambda \bar{\Xi}}^{(N) \bar{\Pi}} a_{0 ; \Pi}^{N}(q),
$$

where the bar denotes the conjugate representation and for the second equality we have used the symmetries of the Clebsch-Gordan coefficients.

Up to now all the equations are valid for finite $k$ and finite $N$. As we have mentioned in section 2.1, in the large $N, k$ limit (2.15) we shall restrict $\Lambda$ and $\Xi$ to the set of those special infinite Young diagrams that can be identified with pairs of finite Young diagrams, see figure 1. Using a free fermion construction, we will show in section 2.4 that the power series expansion of $a_{0 ; \Xi}^{N}(q)$ stabilises in the large $N$ limit to

$$
\begin{aligned}
& a_{0 ; 0}(q)=\lim _{N \rightarrow \infty} a_{0 ; 0}^{N}(q)=\prod_{s=2}^{\infty} \prod_{n=s}^{\infty} \frac{1}{1-q^{n}} \\
& a_{0 ; \Xi}(q)=\lim _{N \rightarrow \infty} a_{0 ; \Xi_{N}}^{N}(q)=\operatorname{ch}_{\Xi_{l}^{t}}\left(U_{0}\right) \operatorname{ch}_{\Xi_{r}^{t}}\left(U_{0}\right) a_{0 ; 0}(q),
\end{aligned}
$$

where $\boldsymbol{\Xi}=\left(\Xi_{l}, \Xi_{r}\right)$ and $U_{0}=U\left(h=\frac{1}{2}\right)$. Note that $\left|a_{0 ; 0}(q)\right|^{2}=Z_{\text {gauge }}(q)$. It follows by a direct calculation (see e.g. eq. (2.7) in [56]) that

$$
\operatorname{Cas}\left(\boldsymbol{\Lambda}_{N}\right)=\operatorname{Cas}\left(\Lambda_{l}\right)+\operatorname{Cas}\left(\Lambda_{r}\right)+\frac{2\left|\Lambda_{l}\right|\left|\Lambda_{r}\right|}{N} .
$$

Thus, for large $N$ the conformal dimensions of the affine primaries behave as

$$
h_{\Lambda_{N}}^{N, k}=\frac{N\left(\left|\Lambda_{l}\right|+\left|\Lambda_{r}\right|\right)}{2(k+N)}+\mathcal{O}\left(\frac{1}{N}\right),
$$

where $\boldsymbol{\Lambda}=\left(\Lambda_{l}, \Lambda_{r}\right)$ is a pair of finite Young diagrams. Hence the exponent of the prefactor in eq. (2.30) becomes in the 't Hooft limit

$$
\lim _{k, N \rightarrow \infty}\left(h_{\boldsymbol{\Lambda}_{N}^{N}}^{N, k}-h_{\Xi_{N}^{N}}^{N, k+1}\right)=\frac{\lambda}{2}(|\boldsymbol{\Lambda}|-|\boldsymbol{\Xi}|),
$$

where we have defined $|\boldsymbol{\Lambda}|=\left|\Lambda_{l}\right|+\left|\Lambda_{r}\right|$ and similarly $|\boldsymbol{\Xi}|$. Thus, the branching functions corresponding to the $W_{\infty}[\lambda]$ modules (2.17) have the explicit form

$$
b_{\boldsymbol{\Lambda} ; \boldsymbol{\Xi}}^{\lambda}(q)=q^{\frac{\lambda}{2}(|\boldsymbol{\Lambda}|-|\Xi|)} a_{0 ; 0}(q) \sum_{\boldsymbol{\Pi} \in \mathbf{Y}} c_{\boldsymbol{\Lambda} \Xi \bar{\Xi}}^{\overline{\bar{\Pi}}} \operatorname{ch}_{\Pi_{l}^{t}}\left(U_{0}\right) \operatorname{ch}_{\Pi_{r}^{t}}\left(U_{0}\right),
$$

where for $\boldsymbol{\Pi}=\left(\Pi_{l}, \Pi_{r}\right)$ the conjugate representations is $\bar{\Pi}=\left(\Pi_{r}, \Pi_{l}\right)$. 
It was argued in [30] that subtracting out the null-states (see the discussion at the end of section 2.2) is equivalent to restricting $\boldsymbol{\Pi}$ in eq. (2.38) to those Young diagrams that satisfy

$$
|\boldsymbol{\Lambda}|+|\boldsymbol{\Xi}|=|\boldsymbol{\Pi}|
$$

Note that this is similar to what happens for the tensor product decomposition for $\mathfrak{s u}(N)$ in the large $N$ limit. For example, in the tensor product of the fundamental and antifundamental representation of $\mathfrak{s u}(N)$, the projector onto the $\mathfrak{s u}(N)$ invariant state is of the form

$$
\sum_{j=1}^{N} \frac{1}{N} e_{j} \otimes e^{j},
$$

where $e_{j}$ and $e^{j}$ are a basis and the dual basis for the fundamental and anti-fundamental representation, respectively. In the large $N$ limit (2.40) vanishes, and the tensor product is no longer completely decomposable. In our case, the analogue of (2.40) are the states where $|\boldsymbol{\Lambda}|+|\boldsymbol{\Xi}|<|\boldsymbol{\Pi}|$, and they vanish in the large $N$ limit as demonstrated (in some simple examples) in [30]. In terms of the Clebsch-Gordan coefficients, (2.39) implies that

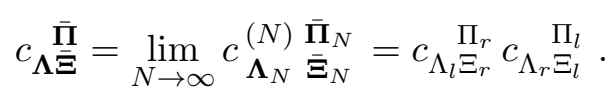

We have furthermore used that the Clebsch-Gordan coefficients on the right hand side stabilise in the large $N$ limit. $^{2}$ Putting everything together we then obtain for the trace over $\mathcal{H}^{\lambda}$

$$
\begin{aligned}
\operatorname{Tr}_{\mathcal{H}^{\lambda}} q^{L_{0}} \bar{q}^{\bar{L}_{0}}=Z_{\text {gauge }} \sum_{\boldsymbol{\Lambda}, \boldsymbol{\Xi}}\left|q^{\frac{\lambda}{2}(|\boldsymbol{\Lambda}|-|\boldsymbol{\Xi}|)} \sum_{\Pi_{l}, \Pi_{r}} c_{\Lambda_{l} \Xi_{r}}{ }^{\Pi_{r}} c_{\Lambda_{r} \Xi_{l}}^{\Pi_{l}} \operatorname{ch}_{\Pi_{l}^{t}}\left(U_{0}\right) \operatorname{ch}_{\Pi_{r}^{t}}\left(U_{0}\right)\right|^{2} \\
=Z_{\text {gauge }} \sum_{\boldsymbol{\Lambda}, \boldsymbol{\Xi}}\left|q^{\frac{\lambda}{2}(|\boldsymbol{\Lambda}|-|\boldsymbol{\Xi}|)} \operatorname{ch}_{\Lambda_{l}^{t}}\left(U_{0}\right) \operatorname{ch}_{\Xi_{r}^{t}}\left(U_{0}\right) \operatorname{ch}_{\Lambda_{r}^{t}}\left(U_{0}\right) \operatorname{ch}_{\Xi_{l}^{t}}\left(U_{0}\right)\right|^{2} \\
=Z_{\text {gauge }} \sum_{\substack{\Lambda_{l}, \Lambda_{r} \\
\Xi_{l}, \Xi_{r}}}\left|\operatorname{ch}_{\Lambda_{l}^{t}}\left(U_{+}\right) \operatorname{ch}_{\Xi_{r}^{t}}\left(U_{-}\right) \operatorname{ch}_{\Lambda_{r}^{t}}\left(U_{+}\right) \operatorname{ch}_{\Xi_{l}^{t}}\left(U_{-}\right)\right|^{2}
\end{aligned}
$$

where we have used that the Clebsch-Gordan coefficients are invariant under taking transposes. This then agrees with (2.25).

\subsection{Free field realisation}

Finally we come to the proof of the two fundamental eqs. (2.34); this is where our analysis differs from [30]. Recall that we can realise $\mathfrak{s u}(N)_{1} \oplus \mathfrak{u}(1)_{N}$ in terms of $N$ free Dirac fermions. Here $\mathfrak{u}(1)_{N}$ is the chiral $\mathfrak{u}(1)$ algebra $\left[J_{m}, J_{n}\right]=N \delta_{m,-n}$ that is extended by two fields of conformal dimension $h=\frac{N}{2}$ and $\mathfrak{u}(1)$-charge $\pm N$, (see [57], section 14.4.4). (Incidentally, $\mathfrak{u}(1)_{N}$ is also the chiral algebra of a compact boson compactified at $R=\sqrt{N}$ where $R=\sqrt{2}$

\footnotetext{
${ }^{2}$ In the theory of symmetric functions the numbers $c_{\Lambda} \Pi$ are known as the Littlewood-Richardson coefficients, (see [54], chapter 1). Essentially, these are the Clebsch-Gordan coefficients of $\mathfrak{g l}(\infty)_{+}$.
} 
describes the self-dual radius, i.e. $\mathfrak{u}(1)_{2} \cong \mathfrak{s u}(2)_{1}$.) The irreducible representations of $\mathfrak{u}(1)_{N}$ are labelled by $l \in \mathbb{Z}_{N}$, and their characters are

$$
\Theta_{l}^{N}(q, w)=\operatorname{Tr}_{l}\left(q^{L_{0}} w^{J_{0}}\right)=\sum_{m \in Z} w^{l+N m} \frac{q^{\frac{1}{2 N}(l+N m)^{2}}}{\prod_{n=1}^{\infty}\left(1-q^{n}\right)} .
$$

On the level of characters, the relation between the free fermion theory and $\mathfrak{s u}(N)_{1} \oplus \mathfrak{u}(1)_{N}$ amounts then to

$$
\prod_{n=0}^{\infty} \prod_{i=1}^{N}\left(1+w v_{i} q^{n+\frac{1}{2}}\right)\left(1+\bar{w} \bar{v}_{i} q^{n+\frac{1}{2}}\right)=\sum_{l=0}^{N-1} \Theta_{l}^{N}(q, w) \operatorname{ch}_{\omega_{l}}^{N, 1}(q, v),
$$

where $\omega_{l}$ is the $l$-th fundamental weight of $\mathfrak{s u}(N), w \in \mathrm{U}(1)$ and $v_{i}$ are the diagonal entries of an element $v$ of the Cartan torus of $\mathrm{SU}(N)$. In terms of the branching functions introduced in eq. (2.31), this then becomes

$$
\prod_{n=0}^{\infty} \prod_{i=1}^{N}\left(1+w v_{i} q^{n+\frac{1}{2}}\right)\left(1+\bar{w} \bar{v}_{i} q^{n+\frac{1}{2}}\right)=\sum_{\Lambda \in Y_{N}} \Theta_{[\Lambda]}^{N}(q, w) a_{0, \Lambda}^{N}(q) \operatorname{ch}_{\Lambda}^{N}(v)
$$

where $[\Lambda] \in \mathbb{Z}_{N}$ denotes the congruence class of an $\mathfrak{s u}(N)$ representation $\Lambda$, (see [57], section 13.1.9). For the following it is more convenient to decompose this partition function into characters of $\mathfrak{u}(N)$ rather then $\mathfrak{s u}(N)$, i.e. to absorb the $w$-dependent factor of $\Theta_{[\Lambda]}^{N}$ into the $\mathfrak{u}(N)$ character as

$$
\operatorname{ch}_{\boldsymbol{\Lambda}_{N}}^{N}(v) w^{\left|\Lambda_{r}\right|-\left|\Lambda_{l}\right|}=\operatorname{ch}_{\Lambda}^{N}(v w)
$$

Recall that $\mathfrak{u}(N)$ representations are parametrised by pairs of Young diagrams $\boldsymbol{\Lambda}=\left(\Lambda_{l}, \Lambda_{r}\right)$, see figure 2; alternatively, we may label them by a single Young diagram $\boldsymbol{\Lambda}_{N}$ together with an integer $|\boldsymbol{\Lambda}|_{-}=\left|\Lambda_{r}\right|-\left|\Lambda_{l}\right|$ determining the $\mathfrak{u}(1)$-charge of the representation. Using (2.46), we can now rewrite (2.45) as

$$
\prod_{n=0}^{\infty} \prod_{i=1}^{N}\left(1+w v_{i} q^{n+\frac{1}{2}}\right)\left(1+\bar{w} \bar{v}_{i} q^{n+\frac{1}{2}}\right)=\sum_{\boldsymbol{\Lambda} \in \mathbf{Y}} d_{\boldsymbol{\Lambda}}^{N}(q) \operatorname{ch}_{\boldsymbol{\Lambda}}^{N}(v w)
$$

where

$$
d_{\boldsymbol{\Lambda}}^{N}(q)=\frac{q^{\frac{1}{2 N}\left(\left|\Lambda_{r}\right|-\left|\Lambda_{l}\right|\right)^{2}}}{\prod_{n=1}^{\infty}\left(1-q^{n}\right)} a_{0, \boldsymbol{\Lambda}_{N}}^{N}(q)
$$

counts the number of $\mathfrak{u}(N)$ tensors $\boldsymbol{\Lambda}$ which appear in the free fermion theory. In the following we shall compute (2.48) combinatorially. Note that the prefactor in the numerator will become irrelevant for $N \rightarrow \infty$.

Let us denote by $\psi^{1}, \ldots, \psi^{N}$ the $N$ Dirac fermions, with $\bar{\psi}^{1}, \ldots, \bar{\psi}^{N}$ their complex conjugates. The vector space whose character is the left-hand-side of (2.47) is spanned by the vectors of the form

$$
\prod_{j=1}^{n_{\bar{\psi}}} \bar{\psi}_{-r_{j}-\frac{1}{2}}^{a_{j}} \prod_{k=1}^{n_{\psi}} \psi_{-s_{k}-\frac{1}{2}}^{b_{k}} \Omega
$$


where $a_{j}, b_{k} \in\{1, \ldots, N\}, r_{j}, s_{k} \in \mathbb{N}_{0}$, and $\Omega$ is the vacuum. These states fall into representations of the two commuting Lie algebras: $\mathfrak{u}(N)$ acting on the indices $a_{j}$ and $b_{k}$; and $\mathfrak{g l}(\infty)_{+}$acting on the mode numbers $r_{j}, s_{k} .{ }^{3}$ The branching function $d_{\boldsymbol{\Lambda}}^{N}$ in (2.47) counts the multiplicity with which the $\mathfrak{u}(N)$ representation $\boldsymbol{\Lambda}$ appears in the Fock space, and because of the commuting $\mathfrak{g l}(\infty)_{+}$action, it will naturally be a character of $\mathfrak{g l}(\infty)_{+}$.

More precisely, a $\mathfrak{u}(N)$ tensor of shape $\boldsymbol{\Lambda}$ appears 'for the first time' (i.e. with multiplicity at most one) in the states of the form (2.49) if $n_{\bar{\psi}}=\left|\Lambda_{l}\right|$ and $n_{\psi}=\left|\Lambda_{r}\right|$. For a given choice of mode numbers $r_{j}$ and $s_{k}$, the multiplicity is precisely one if the $\left\{r_{j}\right\}$ and $\left\{s_{k}\right\}$ define an allowed filling of the Young diagram $\Lambda_{l}^{t}$ and $\Lambda_{r}^{t}$, respectively, where $\Lambda^{t}$ denotes the transposed Young diagram - this just keeps track of the fact that, because of Fermi-Dirac statistics, the product of two identical fermionic modes vanishes. If we sum over all such mode numbers (while keeping $n_{\bar{\psi}}=\left|\Lambda_{l}\right|$ and $n_{\psi}=\left|\Lambda_{r}\right|$ fixed), it follows from (2.22) that the branching function equals

$$
\operatorname{ch}_{\Lambda_{l}^{t}}\left(U_{0}\right) \operatorname{ch}_{\Lambda_{r}^{t}}\left(U_{0}\right)
$$

where $U_{0}=U\left(h=\frac{1}{2}\right)$.

In order to complete the argument we only need to count the multiplicities with which the $\mathfrak{u}(N)$ representations $\boldsymbol{\Lambda}$ appear. As we have explained above, a given $\boldsymbol{\Lambda}$ appears 'for the first time' if $n_{\bar{\psi}}=\left|\Lambda_{l}\right|$ and $n_{\psi}=\left|\Lambda_{r}\right|$. However, it will continue to appear if $n_{\bar{\psi}}=\left|\Lambda_{l}\right|+m$ and $n_{\psi}=\left|\Lambda_{r}\right|+m$ with $m \in \mathbb{N}$, i.e. the state can be a product of a state with minimal number of factors, times a $\mathfrak{u}(N)$ invariant state. Thus we need to count also the $\mathfrak{u}(N)$ invariants; according to the first fundamental theorem of classical invariant theory (see e.g. [58]), all $\mathfrak{u}(N)$ invariant states are linear combinations of the 'basic' ones

$$
\prod_{r, s=0}^{\infty}\left(\sum_{a} \bar{\psi}_{-r-\frac{1}{2}}^{a} \psi_{-s-\frac{1}{2}}^{a}\right)^{M_{r s}}
$$

where only finitely many multiplicities $M_{r s}$ are non-zero. Note that not all of these states are non-trivial; indeed, (2.51) vanishes if

$$
\sum_{r} M_{r s}>N \quad \text { or } \quad \sum_{s} M_{r s}>N
$$

Furthermore, the states corresponding to different choices of $\left\{M_{r s}\right\}$ are not all linearly independent; for example, for $N=1$ the two states whose non-zero multiplicities are $\left\{M_{00}=1, M_{11}=1\right\}$ and $\left\{M_{01}=1, M_{10}=1\right\}$ are in fact linearly dependent. If we ignore these issues we can easily count the invariant tensors as

$$
d_{0}(q)=\prod_{r, s=0}^{\infty} \sum_{M_{r s}=0}^{\infty} q^{(r+s+1) M_{r s}}=\prod_{r, s=0}^{\infty} \frac{1}{1-q^{r+s+1}}=\prod_{s=1}^{\infty} \prod_{n=s}^{\infty} \frac{1}{1-q^{n}} .
$$

This result is exact in the $N \rightarrow \infty$ limit because for finite $N$ the overcounting starts at order $q^{N+1}$ with the state

$$
\left(\sum_{a} \bar{\psi}_{-\frac{1}{2}}^{a} \psi_{-\frac{1}{2}}^{a}\right)^{N+1}
$$

\footnotetext{
${ }^{3}$ Note that the action of $\mathfrak{g l}(\infty)_{+}$on the modes of $\psi^{a}$, and on the modes of $\bar{\psi}^{a}$ is in both cases the fundamental representation of $\mathfrak{g l}(\infty)_{+}$.
} 
Combining this result with (2.50), and ignoring the subtlety that the product of a nonvanishing scalar and a state of the form (2.49) in some representation $\boldsymbol{\Lambda}$ can also vanish — again this can be ignored in the $N \rightarrow \infty$ limit - then leads precisely to (2.34). (Recall that the relation between $a_{0, \boldsymbol{\Lambda}}^{N}$ and $d_{\boldsymbol{\Lambda}}^{N}$ is given in (2.48).)

\section{Supersymmetric duality}

In the following we want to generalise the above argument to the supersymmetric setting. We begin by reviewing the structure of the supersymmetric higher spin theory.

\subsection{Higher spin supergravity}

The $\mathcal{N}=2$ supersymmetric higher spin supergravity theory of Prokushkin and Vasiliev [22, 23] has two (real) bosonic gauge fields of each spin $s=2,3, \ldots$, together with a single current of spin $s=1$. In addition there are two (real) fermionic gauge fields for each spin $s=\frac{3}{2}, \frac{5}{2}, \ldots$. As in the bosonic case above, the structure of the theory depends on a real parameter $0 \leq \lambda \leq 1$. However, this parameter does not affect the quadratic part of the action, and the total 1-loop contribution of the gauge fields equals

$$
\mathcal{Z}_{\text {gauge }}=Z_{\text {gauge }}^{1} \prod_{s=2}^{\infty}\left(Z_{\text {gauge }}^{s} Z_{\text {gaugino }}^{s-\frac{1}{2}}\right)^{2},
$$

where the contribution of a real gauge field of half-integer spin $s$

$$
Z_{\text {gaugino }}^{s}=\prod_{n=s-\frac{1}{2}}^{\infty}\left|1+q^{n+\frac{1}{2}}\right|^{2}
$$

was calculated in [44]. We have assumed here that these half-integer spin gauge fields have anti-periodic boundary conditions around the thermal circle; from the dual CFT point of view, we shall therefore only consider the NS-sector. We also note that we can write $\mathcal{Z}_{\text {gauge }}$ in a manifestly supersymmetric way as

$$
\mathcal{Z}_{\text {gauge }}=\prod_{s=1}^{\infty} \mathcal{Z}_{\text {gauge }}^{s}, \quad \text { where } \quad \mathcal{Z}_{\text {gauge }}^{s}=Z_{\text {gauge }}^{s}\left(Z_{\text {gauge }}^{s+\frac{1}{2}}\right)^{2} Z_{\text {gauge }}^{s+1},
$$

with $\mathcal{Z}_{\text {gauge }}^{s}$ the contribution of the $\mathcal{N}=2$ gauge multiplet of integer spin $s$.

While the parameter $\lambda$ from above does not appear in the quadratic action for the gauge fields, it does determine the mass of the fields in the allowed matter multiplets. In the supersymmetric case, each matter multiplet consists of a complex scalar field of mass

$$
M_{\lambda}^{2}=-1+\lambda^{2}
$$

two Dirac fermions of mass

$$
m^{2}=\left(\lambda-\frac{1}{2}\right)^{2},
$$




$$
\begin{aligned}
& M_{\lambda}(+) \quad m(-) \quad M_{1-\lambda}(-) \\
& \left(\frac{1}{2}+\frac{\lambda}{2}, \frac{1}{2}+\frac{\lambda}{2}\right) \quad \stackrel{Q^{ \pm}, \tilde{Q}^{ \pm}}{\longleftrightarrow} \quad 2 \times \begin{array}{l}
\left(\frac{\lambda}{2}, \frac{1}{2}+\frac{\lambda}{2}\right) \\
\left(\frac{1}{2}+\frac{\lambda}{2}, \frac{\lambda}{2}\right)
\end{array} \stackrel{Q^{ \pm}, \tilde{Q}^{ \pm}}{\longleftrightarrow} \quad\left(\frac{\lambda}{2}, \frac{\lambda}{2}\right) \\
& \begin{array}{lll}
M_{\lambda}(-) & m(+) & M_{1-\lambda}(+)
\end{array} \\
& \left(\frac{1}{2}-\frac{\lambda}{2}, \frac{1}{2}-\frac{\lambda}{2}\right) \quad \stackrel{Q^{ \pm}, \tilde{Q}^{ \pm}}{\longleftrightarrow} 2 \times \begin{array}{l}
\left(\frac{1}{2}-\frac{\lambda}{2}, 1-\frac{\lambda}{2}\right) \\
\left(1-\frac{\lambda}{2}, \frac{1}{2}-\frac{\lambda}{2}\right)
\end{array} \quad \stackrel{Q^{ \pm}, \tilde{Q}^{ \pm}}{\longleftrightarrow} \quad\left(1-\frac{\lambda}{2}, 1-\frac{\lambda}{2}\right)
\end{aligned}
$$

Figure 3. Conformal dimensions of the scalar and spinor fields in the two short $\mathcal{N}=2$ complex supermultiplets. Here $Q^{ \pm}$and $\tilde{Q}^{ \pm}$are the left- and right-moving $\mathcal{N}=2$ supercharges in the CFT. Since the representation is short, one of the two supercharges of each chirality always acts trivially. The Dirac fermions have multiplicity 2 since the scalar fields are complex.

as well as a complex scalar of mass $M_{1-\lambda}$. These are actually short $\mathcal{N}=2$ complex supermultiplets, and the corresponding states in the dual CFT are $\mathcal{N}=2$ chiral primaries. ${ }^{4}$

The propagation of the free (massive) scalar or spinor fields on $\mathrm{AdS}_{3}$ is unambiguously fixed by the respective equations of motion provided one specifies the asymptotic behaviour of the fields at the boundary, i.e. the conformal dimensions of the dual superconformal fields. For the fields in the above mass windows, there are two natural boundary conditions one may choose, and we shall refer to them as the $( \pm)$ quantisations; for the scalar fields the relevant dual conformal dimensions are again $(h, h)$ with $\Delta=2 h$ being given by $(2.5)$, while for a massive Dirac fermion the relevant conformal dimensions are $\left(h+\frac{1}{2}, h\right)$ and $\left(h, h+\frac{1}{2}\right)$ with $\Delta=2 h+\frac{1}{2}$ given by

$$
\Delta_{+}^{F}=\frac{3}{2}-\lambda, \quad \Delta_{-}^{F}=\lambda+\frac{1}{2} \quad \text { i.e. } \quad h_{+}^{F}=\frac{1}{2}(1-\lambda), \quad h_{-}^{F}=\frac{\lambda}{2} .
$$

The contribution of the complex scalar field with $\Delta=2 h$ to the 1-loop partition function is again given by (2.3), while that of a Dirac fermion with conformal dimensions $\left(h+\frac{1}{2}, h\right)$ and $\left(h, h+\frac{1}{2}\right)$ is [44]

$$
Z_{\text {spinor }}^{\Delta}=\prod_{m, n=0}^{\infty}\left(1+q^{h+\frac{1}{2}+m} \bar{q}^{h+n}\right)\left(1+q^{h+m} \bar{q}^{h+\frac{1}{2}+n}\right) .
$$

Notice that supersymmetry determines unambiguously the quantisation of all fields in a supermultiplet in terms of, for instance, the quantisation of the scalar $M_{\lambda}^{2}$. More precisely, the two scalars $M_{\lambda}^{2}$ and $M_{1-\lambda}^{2}$ are quantised in an opposite fashion, while the fermions are quantised, due to our conventions (3.5), in the same way as $M_{1-\lambda}^{2}$. This is illustrated in figure 3.

The complete matter spectrum of the higher spin theory of [44] consists of two such $\mathcal{N}=2$ multiplets that are quantised again in the opposite fashion; altogether the 1-loop

\footnotetext{
${ }^{4}$ Notice that in 3 Euclidean dimensions the action of a supercharge on a real scalar field gives a Dirac fermion.
} 
partition function $\mathcal{Z}_{1 \text {-loop }}^{\lambda}$ of this theory is therefore

$$
\mathcal{Z}_{1 \text {-loop }}^{\lambda}=\mathcal{Z}_{\text {gauge }} \times Z_{\text {scalars }} \times Z_{\text {spinors }}
$$

where $\mathcal{Z}_{\text {gauge }}$ was defined in (3.3), and the scalar and spinor contributions are

$$
\begin{aligned}
& Z_{\text {scalars }}=Z_{\text {scalar }}^{\Delta_{+}^{B}(\lambda)} Z_{\text {scalar }}^{\Delta_{-}^{B}(\lambda)} Z_{\text {scalar }}^{\Delta_{+}^{B}(1-\lambda)} Z_{\text {scalar }}^{\Delta_{-}^{B}(1-\lambda)} \\
& Z_{\text {spinors }}=\left(Z_{\text {spinor }}^{\Delta_{+}^{F}} Z_{\text {spinor }}^{\Delta_{-}^{F}}\right)^{2}
\end{aligned}
$$

Note that the total partition function is invariant under $\lambda \mapsto 1-\lambda$. In terms of $\mathcal{N}=2$ supermultiplets, we have

$$
\mathcal{Z}_{1 \text {-loop }}^{\lambda}=\mathcal{Z}_{\text {gauge }} \times \mathcal{Z}_{\text {matter }}^{\lambda,+} \times \mathcal{Z}_{\text {matter }}^{\lambda,-}
$$

where the first two factors denote the contribution of the two $\mathcal{N}=2$ matter multiplets from above

$$
\begin{aligned}
& \mathcal{Z}_{\text {matter }}^{\lambda,+}=Z_{\text {scalar }}^{\Delta_{+}^{B}(\lambda)} Z_{\text {scalar }}^{\Delta_{-}^{B}(1-\lambda)}\left(Z_{\text {spinor }}^{\Delta_{-}^{F}}\right)^{2}=Z_{\text {scalar }}^{\lambda}\left(Z_{\text {spinor }}^{\frac{1}{2}+\lambda}\right)^{2} Z_{\text {scalar }}^{1+\lambda} \\
& \mathcal{Z}_{\text {matter }}^{\lambda,-}=Z_{\text {scalar }}^{\Delta_{+}^{B}(1-\lambda)} Z_{\text {scalar }}^{\Delta_{-}^{B}(\lambda)}\left(Z_{\text {spinor }}^{\Delta_{+}^{F}}\right)^{2}=Z_{\text {scalar }}^{1-\lambda}\left(Z_{\text {spinor }}^{\frac{3}{2}-\lambda}\right)^{2} Z_{\text {scalar }}^{2-\lambda}=\mathcal{Z}_{\text {matter }}^{1-\lambda,+} .
\end{aligned}
$$

Expanding them out as above, we then have explicitly

$$
\mathcal{Z}_{\text {matter }}^{\lambda,+}=\prod_{m, n=0}^{\infty} \frac{\left(1+q^{\frac{\lambda}{2}+\frac{1}{2}+m} \bar{q}^{\frac{\lambda}{2}+n}\right)^{2}\left(1+q^{\frac{\lambda}{2}+m} \bar{q}^{\frac{\lambda}{2}+\frac{1}{2}+n}\right)^{2}}{\left(1-q^{\frac{\lambda}{2}+m} \bar{q}^{\frac{\lambda}{2}+n}\right)^{2}\left(1-q^{\frac{\lambda}{2}+\frac{1}{2}+m} \bar{q}^{\frac{\lambda}{2}+\frac{1}{2}+n}\right)^{2}} .
$$

\subsection{The superconformal coset}

It was proposed in [44] that the above higher spin theory is dual to the 't Hooft like limit of a family of minimal $\mathcal{N}=2$ superconformal coset theories. In this section we want to review the relevant superconformal field theories.

Recall that we can associate to each bosonic affine algebra $\mathfrak{s u}(N)_{k}$ an $\mathcal{N}=1$ supersymmetric affine algebra $\mathfrak{s u}(N)_{k+N}^{1}$; the latter is actually isomorphic to the direct sum of the bosonic algebra $\mathfrak{s u}(N)_{k}$ together with $\operatorname{dim}(\mathfrak{s u}(N))$ free Majorana fermions. In analogy to this, we also denote by $\mathfrak{u}(1)_{k}^{1}$ the direct sum of $\mathfrak{u}(1)_{k}$ and (the chiral superalgebra of) a single Majorana fermion.

The cosets that are relevant for us are then

$$
\mathcal{W}_{N, k}=\frac{\mathfrak{s u}(N+1)_{k+N+1}^{1}}{\mathfrak{s u}(N)_{k+N+1}^{1} \oplus \mathfrak{u}(1)_{\kappa}^{1}},
$$

where $\kappa=N(N+1)(k+N+1)$ is the 'level' of the $\mathfrak{u}(1)$ algebra (as defined above eq. (2.43)). They are manifestly $\mathcal{N}=1$ supersymmetric, but according to Kazama and Suzuki $[45,46]$, 
the actual chiral algebra contains the $\mathcal{N}=2$ superconformal algebra. Geometrically, this is a consequence of the fact that the coset (3.13) is associated to the homogeneous space

$$
\mathbb{C P}^{N}=\frac{\mathrm{U}(N+1)}{\mathrm{U}(N) \times \mathrm{U}(1)}
$$

which is actually a Hermitian symmetric space, i.e. possesses a complex structure. We should also mention in passing that (3.13) coincides with the Drinfel'd-Sokolov reduction of the affine superalgebra $\operatorname{sl}(N+1 \mid N)_{k_{\mathrm{DS}}}$ at level [59]

$$
k_{\mathrm{DS}}=-1+\frac{1}{k+n+1} .
$$

Given that the $\mathcal{N}=1$ superconformal algebras are actually isomorphic to direct sums of the corresponding bosonic subalgebras and free Majorana fermions, we can reformulate the bosonic subalgebra of $\mathcal{W}_{N, k}$ in (3.13) as

$$
\mathcal{W}_{N, k}^{(0)}=\frac{\mathfrak{s u}(N+1)_{k} \oplus \mathfrak{s o}(2 N)_{1}}{\mathfrak{s u}(N)_{k+1} \oplus \mathfrak{u}(1)_{\kappa}}
$$

where $\mathfrak{s o}(2 N)_{1}$ is the bosonic algebra associated to the $2 N$ free Majorana fermions that survive after subtracting from the $N^{2}+2 N$ free fermions of the numerator in (3.13) the $N^{2}$ free fermions of the denominator. The central charge of the coset algebra $\mathcal{W}_{N, k}$ is therefore

$$
c=(N-1)+\frac{k N(N+2)}{k+N+1}-\frac{(k+1)\left(N^{2}-1\right)}{k+N+1}=\frac{3 k N}{k+N+1} .
$$

In the following we shall mostly use the bosonic coset description (3.16); note that this description contains implicitly the supersymmetry generators as long as we describe the $\mathfrak{s o}(2 N)_{1}$ algebra in terms of $2 N$ free Majorana fermions.

We shall also need to understand how the denominator of (3.16) is embedded into the numerator. The embedding of $\mathfrak{s u}(N) \oplus \mathfrak{u}(1)$ into the first factor (i.e. into $\mathfrak{s u}(N+1)$ ) is determined by the usual embedding of $\mathrm{SU}(N) \times \mathrm{U}(1) \hookrightarrow \mathrm{SU}(N+1)$,

$$
\imath_{1}(v, w)=\left(\begin{array}{cc}
w^{N} & 0 \\
0 & \bar{w} v
\end{array}\right) \in \mathrm{SU}(N+1),
$$

where $v \in \mathrm{SU}(N)$ and $w \in \mathrm{U}(1)$. Let us denote by $K \in \mathfrak{s u}(N+1)$ the image of the $\mathfrak{u}(1)$ Lie algebra generator (i.e. $K$ is the diagonal matrix with entries $(N,-1, \ldots,-1)$ ); its OPE is then of the form

$$
K\left(z_{1}\right) K\left(z_{2}\right)=\frac{k N(N+1)}{\left(z_{1}-z_{2}\right)^{2}}+\mathcal{O}(1) .
$$

In order to understand the embedding into the $\mathfrak{s o}(2 N)$ factor, recall that we can think of $\mathfrak{s o}(2 N)$ as the Lie algebra of the Lie group $\mathrm{SO}(N, N)$ of $2 N \times 2 N$ matrices $M$ satisfying $M G M^{t}=G$ with

$$
G=\left(\begin{array}{cc}
0 & \mathbb{1}_{\mathrm{N}} \\
\mathbb{1}_{\mathrm{N}} & 0
\end{array}\right)
$$


We then embed $\mathrm{SU}(N) \times \mathrm{U}(1) \hookrightarrow \mathrm{SO}(N, N)$ (the scaling of the $\mathrm{U}(1)$ embedding relative to (3.18) is fixed by $\mathcal{N}=1$ supersymmetry, see (3.44) below) as

$$
\imath_{2}(v, w)=\left(\begin{array}{cc}
\bar{w}^{(N+1)} v & 0 \\
0 & w^{(N+1)} \bar{v}
\end{array}\right) \in \mathrm{SO}(N, N),
$$

where $\bar{v}$ denotes the complex conjugate matrix to $v \in \mathrm{SU}(N)$. Again we denote by $j \in$ $\mathfrak{s o}(2 N)$ the image of the $\mathfrak{u}(1)$ Lie algebra generator (whose first $N$ diagonal entries are $-(N+1)$, with the remaining diagonal entries being equal to $N+1)$; its OPE is then

$$
j\left(z_{1}\right) j\left(z_{2}\right)=\frac{N(N+1)^{2}}{\left(z_{1}-z_{2}\right)^{2}}+\mathcal{O}(1) .
$$

Together with (3.19) it then follows that the current

$$
J=\frac{1}{k+N+1}\left(K-\frac{k}{N+1} j\right)
$$

is primary with respect to the denominator algebra; it therefore describes a $\mathfrak{u}(1)$-current of the coset algebra $\mathcal{W}_{N, k}$. It can be identified with the $\mathfrak{u}(1)$-current of the $\mathcal{N}=2$ superconformal subalgebra, and with the above normalisation of $K$ and $j$ it is canonically normalised so that

$$
J\left(z_{1}\right) J\left(z_{2}\right)=\frac{c}{3\left(z_{1}-z_{2}\right)^{2}}+\mathcal{O}(1)
$$

where $c$ is given in (3.17).

The irreducible representations of $\mathcal{W}_{N, k}$ can again be described in the usual manner. Let us denote by NS the Neveu-Schwarz representation of the $2 N$ Majorana fermions. (From the point of view of $\mathfrak{s o}(2 N)_{1}$, NS is therefore the direct sum of the vacuum and the vector representation.) For any integrable representation $\Lambda \in Y_{N+1, k}$ of $\mathfrak{s u}(N+1)_{k}$ we then consider the decomposition of the tensor product

$$
\Lambda \otimes \mathrm{NS}=\bigoplus_{\Xi, l}(\Lambda ; \Xi, l) \otimes \Xi \otimes l
$$

with respect to $\mathfrak{s u}(N)_{k+1} \oplus \mathfrak{u}(1)_{\kappa}$. Here $\Xi \in Y_{N, k+1}$ labels the representations of $\mathfrak{s u}(N)_{k+1}$, while $l \in \mathbb{Z}_{\kappa}$ describes the representations of $\mathfrak{u}(1)_{\kappa}$. In order to understand which representations of $\mathfrak{s u}(N)_{k+1} \oplus \mathfrak{u}(1)_{\kappa}$ appear in this decomposition, let us write $\Lambda$ and $\Xi$ in terms of the usual orthogonal basis as

$$
\Lambda=\sum_{j=0}^{N} \Lambda_{j} \varepsilon_{j}-\frac{|\Lambda|}{N+1} \sum_{j=0}^{N} \varepsilon_{j} \quad \text { and } \quad \Xi=\sum_{j=1}^{N} \Xi_{j} \varepsilon_{j}-\frac{|\Xi|}{N} \sum_{j=1}^{N} \varepsilon_{j},
$$

where $\Lambda_{j}$ and $\Xi_{j}$ are the number of boxes in the $j$ 'th row of $\Lambda$ and $\Xi$, respectively. (For the case of $\mathfrak{s u}(N+1)$ the first row is the zero'th row, while for $\mathfrak{s u}(N)$, the rows are labelled by $1, \ldots, N$.) Given the structure of the embedding (3.18), the weight of the $\mathfrak{u}(1)_{\kappa}$ representation labelled by $l$ in eq. (3.25) is then of the form

$$
\omega_{l}=\frac{l}{N(N+1)}\left(N \varepsilon_{0}-\sum_{j=1}^{N} \varepsilon_{j}\right) \quad \text { so that } \quad \omega_{l}(K)=l,
$$


while $\omega_{l}$ vanishes on all generators of $\mathfrak{s u}(N)_{k+1}$ under the embedding $\imath_{1}$. The root lattice of $\mathfrak{s u}(N+1)$ is generated by the vectors $\varepsilon_{j}$, and hence the selection rule that $\Lambda-\Xi-\omega_{l}$ lies in the root lattice of $\mathfrak{s u}(N+1)$ simply means that the coefficients of all $\varepsilon_{j}$ are integer; for $j \neq 0$ this is precisely the condition that

$$
\frac{|\Lambda|}{N+1}-\frac{|\Xi|}{N}-\frac{l}{N(N+1)} \equiv 0 \bmod 1
$$

and it is easy to see that then also the coefficient of $\varepsilon_{0}$ is integer. Note that (3.28) determines $l$ in terms of $\Lambda$ and $\Xi$ only modulo $N(N+1)$; since $l$ is defined modulo $\kappa=N(N+1)(N+$ $k+1)$, it is not completely fixed by $(3.28) .{ }^{5}$

The multiplicity spaces labelled by $(\Lambda ; \Xi, l)$ satisfying (3.28) then define representations of $\mathcal{W}_{N, k}$. In fact, all representations of $\mathcal{W}_{N, k}$ can be described in this manner. However, not all triplets $(\Lambda ; \Xi, l)$ lead to inequivalent representations; the relevant identification rules are worked out in [51].

The character of $2 N$ Neveu-Schwarz Majorana fermions equals

$$
\theta(q, u)=\operatorname{tr}_{\mathrm{NS}} q^{L_{0}} u=\prod_{n=0}^{\infty} \prod_{i=1}^{N}\left(1+u_{i} q^{n+\frac{1}{2}}\right)\left(1+\bar{u}_{i} q^{n+\frac{1}{2}}\right),
$$

where $u$ is an $\operatorname{SO}(N, N)$ group element with eigenvalues $\left\{u_{i}, \bar{u}_{i}\right\}_{i=1}^{N}$. Together with the affine characters defined in (2.11) and (2.43) we then have the identity

$$
\operatorname{ch}_{\Lambda}^{N+1, k}\left(q, \imath_{1}(v, w)\right) \theta\left(q, \iota_{2}(v, w)\right)=\sum_{\Xi, l} b_{\Lambda ; \Xi, l}^{N, k}(q) \operatorname{ch}_{\Xi}^{N, k+1}(q, v) \Theta_{l}^{\kappa}(q, w)
$$

where

$$
b_{\Lambda ; \Xi, l}^{N, k}(q)=\operatorname{tr}_{(\Lambda ; \Xi, l)} q^{L_{0}}
$$

is again the coset character.

The simplest CFT is as before the charge conjugation theory whose full space of states is of the form

$$
\mathcal{H}_{s}^{N, k}=\bigoplus_{[\Lambda ; \Xi, l]}[\Lambda ; \Xi, l] \otimes \overline{[\Lambda ; \Xi, l]}
$$

where $[\Lambda ; \Xi, l]$ denotes again the equivalence classes of coset representations. The corresponding torus partition function

$$
\mathcal{Z}^{N, k}(q)=\left|q^{-\frac{c}{24}}\right|^{2} \sum_{[\Lambda ; \Xi, l]}\left|b_{\Lambda ; \Xi, l}^{N, k}(q)\right|^{2}
$$

is then modular invariant with respect to the appropriate modular group (namely the congruence subgroup that is generated by $S$ and $T^{2}$ ). Here we have restricted ourselves to the (unprojected) NS-NS sector. The R-sector representations do not, in any case, contribute to the perturbative spectrum in the 't Hooft limit since their conformal dimensions are proportional to $c$ (which goes to infinity in the limit).

\footnotetext{
${ }^{5}$ The level of the $\mathfrak{u}(1)$ algebra is the central term in the current-current OPE where the current has been normalised so that the spectrum of its zero mode consists of the integers. In our case the correctly normalised current is $K+j$, and the level can then be read off from (3.19) and (3.22).
} 


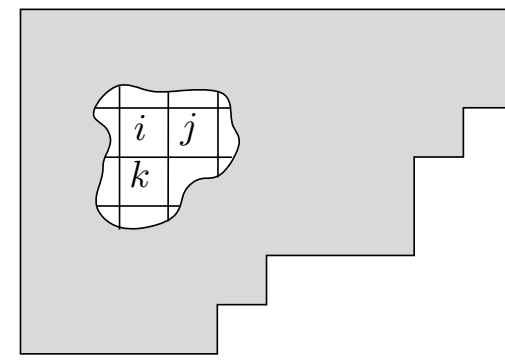

$i \leq j, k$

$i<j$ if $i$ and $j$ are odd

$i<k$ if $i$ and $k$ are even

Figure 4. A supertableau of shape $\Lambda$ and type $\mathfrak{g l}(\infty \mid \infty)_{+}$is a filling of the boxes of a Young diagram $\Lambda$ with elements from $\mathbb{N}_{0}$ such that the entries of the boxes are ordered as indicated in the figure.

\subsection{The duality}

As was already mentioned above, it was proposed in [44] that the higher spin theory of section 3.1 is dual to the large $N, k$ limit (2.15) of the above $\mathcal{N}=2$ minimal model superconformal field theories. In order to define the limit, we restrict, as in the bosonic case of section 2 , the spectrum of (3.32) to those representations $(\Lambda ; \Xi, l)$ for which both $\Lambda$ and $\Xi$ can be labelled by pairs of Young diagrams $\boldsymbol{\Lambda}$ and $\boldsymbol{\Xi}$ as in figure 1. We want to show in the following that with this restriction (and after removing the relevant null-vectors, see below) the partition functions between the two descriptions agree. This provides again very non-trivial evidence in favour of this duality.

\subsubsection{The higher spin partition function}

Let us begin by rewriting the higher spin partition function (3.9) as in the bosonic case, see eq. (2.25), except that now the relevant algebra is $\mathfrak{g l}(\infty \mid \infty)_{+}$, rather than $\mathfrak{g l}(\infty)_{+}$. In order to do so we need to fix some conventions.

Recall that, as a vector space, the algebras $\mathfrak{g l}(\infty)_{+}$and $\mathfrak{g l}(\infty \mid \infty)_{+}$are isomorphic. The only difference is that for the superalgebra $\mathfrak{g l}(\infty \mid \infty)_{+}$we distinguish between the bosonic generators $E_{i j}$ for which $i+j$ is even, and the fermionic generators $E_{i j}$ for which $i+j$ is odd. Correspondingly we then define commutation and anti-commutation relations. It is clear from this description that we have again a representation of $\mathfrak{g l}(\infty \mid \infty)_{+}$on $\mathbb{C}^{\mathrm{N}_{0}}$.

The tensor products of this fundamental representation are completely decomposable into irreducible representations, and these are again labelled by Young diagrams [60, 61]. In order to describe the associated character of $\mathfrak{g l}(\infty \mid \infty)_{+}$, we need to introduce supertableaux. A supertableau is a filling of the Young diagram $\Lambda$ by elements from $\mathbb{N}_{0}$, where the entries do not decrease along rows and columns, and the direction in which they strictly increase depends on the parity of the corresponding entries; the precise rule is explained in figure 4. We can label the basis elements of $\Lambda$ by the different Young supertableaux $T \in \mathrm{STab}_{\Lambda}$ of shape $\Lambda$, and the weight of $T$ is the sum of the fundamental weights $\epsilon_{i}$ associated to $T$, i.e. $\operatorname{wt}(T)=\sum_{i \in T} \epsilon_{i}$. (Note that the Cartan subalgebra of $\mathfrak{g l}(\infty \mid \infty)_{+}$can again be taken to consist of the diagonal matrices, and $\epsilon_{i}$ is then as before defined by eq. (2.18).) The supercharacter of the $\mathfrak{g l}(\infty \mid \infty)_{+}$representation labelled 
by $\Lambda$ is then

$$
\operatorname{sch}_{\Lambda}\left(e^{H}\right)=\sum_{T \in \operatorname{STab}_{\Lambda}} e^{\mathrm{wt}(T)(H)} \prod_{j \in T}(-1)^{j} .
$$

The generalisation of eq. (2.23) that is relevant for us is now

$$
\begin{aligned}
\frac{1}{\operatorname{sdet}\left(1-\mathcal{U}(h) \otimes \mathcal{U}(h)^{*}\right)} & =\prod_{m, n=0}^{\infty} \frac{\left(1+q^{h+\frac{1}{2}+m} \bar{q}^{h+n}\right)\left(1+q^{h+m} \bar{q}^{h+\frac{1}{2}+n}\right)}{\left(1-q^{h+m} \bar{q}^{h+n}\right)\left(1-q^{h+\frac{1}{2}+m} \bar{q}^{h+\frac{1}{2}+n}\right)} \\
& =\sum_{\Lambda} \operatorname{sch}_{\Lambda}(\mathcal{U}(h)) \operatorname{sch}_{\Lambda}\left(\mathcal{U}(h)^{*}\right)
\end{aligned}
$$

where sdet denotes the superdeterminant, and $\mathcal{U}(h)$ is a $\mathrm{GL}(\infty \mid \infty)_{+}$diagonal matrix with matrix elements

$$
\mathcal{U}(h)_{j j}=(-1)^{j} q^{h+\frac{j}{2}} .
$$

On these group elements the supercharacter reads explicitly

$$
\operatorname{sch}_{\Lambda}(\mathcal{U}(h))=\sum_{T \in \mathrm{STab}_{\Lambda}} \prod_{i \in T} q^{h+\frac{i}{2}}
$$

since the parity signs in eq. (3.36) cancel against those in eq. (3.34). Using exactly the same arguments as for the bosonic case, see eq. (2.24), this allows us to write the partition function (3.9) (see in particular (3.12)) in the form

$$
\mathcal{Z}_{\text {1-loop }}^{\lambda}=\mathcal{Z}_{\text {gauge }} \sum_{\Lambda_{l}, \Lambda_{r}, \Xi_{l}, \Xi_{r}}\left|\operatorname{sch}_{\Lambda_{l}}\left(\mathcal{U}_{+}\right) \operatorname{sch}_{\Lambda_{r}}\left(\mathcal{U}_{+}\right) \operatorname{sch}_{\Xi_{l}}\left(\mathcal{U}_{-}\right) \operatorname{sch}_{\Xi_{r}}\left(\mathcal{U}_{-}\right)\right|^{2}
$$

where $\mathcal{U}_{+}=\mathcal{U}\left(h=\frac{\lambda}{2}\right)$ and $\mathcal{U}_{-}=\mathcal{U}\left(h=\frac{1-\lambda}{2}\right)$.

\subsubsection{The superconformal partition function}

Now we come to the CFT partition function in the 't Hooft limit. Using the form of (3.18), we can express the character of $\mathfrak{s u}(N+1)_{k}$ in the large $k$ limit as

$$
\operatorname{ch}_{\Lambda}^{N+1, k}\left(q, \iota_{1}(v, w)\right)=\frac{q_{\Lambda}^{h_{\Lambda}^{N+1, k}}\left[\operatorname{ch}_{\Lambda}^{N+1}\left(\imath_{1}(v, w)\right)+\mathcal{O}\left(q^{k-\Lambda_{1}+1}\right)\right]}{\prod_{n=1}^{\infty}\left(1-q^{n}\right)^{N} \prod_{i \neq j=0}^{N}\left(1-v_{i} \bar{v}_{j} q^{n}\right)},
$$

where $\left\{v_{i}\right\}_{i=1}^{N}$ are the eigenvalues of $v \in \mathrm{SU}(N)$ and we have defined $v_{0}=w^{N+1}$. For the other characters in (3.30) we have similarly

$$
\begin{aligned}
\operatorname{ch}_{\Xi}^{N, k+1}(q, v) & =\frac{q^{h_{\Xi}^{N, k+1}}\left[\operatorname{ch}_{\Xi}^{N}(v)+\mathcal{O}\left(q^{k-\Xi_{1}+2}\right)\right]}{\prod_{n=1}^{\infty}\left(1-q^{n}\right)^{N-1} \prod_{i \neq j=1}^{N}\left(1-v_{i} \bar{v}_{j} q^{n}\right)} \\
\Theta_{l}^{\kappa}(q, w) & =\frac{q^{h_{l}^{\kappa}}}{\prod_{n=1}^{\infty}\left(1-q^{n}\right)}\left[w^{l}+\mathcal{O}\left(q^{\frac{\kappa}{2}-|l|}\right)\right], \quad h_{l}^{\kappa}=\frac{l^{2}}{2 \kappa} .
\end{aligned}
$$

If we define the leading term of the coset character via

$$
b_{\Lambda ; \Xi, l}^{N, k}(q)=q^{h_{\Lambda}^{N+1, k}-h_{\Xi}^{N, k+1}-h_{l}^{\kappa}}\left[a_{\Lambda ; \Xi, l}^{N}(q)+\mathcal{O}\left(q^{k-\Lambda_{1}+1}\right)+\mathcal{O}\left(q^{k-\Xi_{1}+2}\right)\right],
$$


it follows from eq. (3.30) that we have the $k$-independent identity

$$
\operatorname{ch}_{\Lambda}^{N+1}\left(\imath_{1}(v, w)\right) \vartheta\left(q, \imath_{2}(v, w)\right)=\sum_{\Xi, l} a_{\Lambda ; \Xi, l}^{N}(q) \operatorname{ch}_{\Xi}^{N}(v) w^{l},
$$

where the sum runs over all $\Xi \in Y_{N}$ and $l$ must obey the selection rule (3.28). Note that the denominators of (3.39), (3.40) and (3.41) cancel among each other, except for the factors with $i=0$ or $j=0$ in (3.39); because of (3.21), these combine with the contribution of the $N$ Dirac fermions from $\theta$ (see (3.29)) to the supersymmetric combination

$$
\vartheta(q, u)=\prod_{n=0}^{\infty} \prod_{i=1}^{N} \frac{\left(1+u_{i} q^{n+\frac{1}{2}}\right)\left(1+\bar{u}_{i} q^{n+\frac{1}{2}}\right)}{\left(1-u_{i} q^{n+1}\right)\left(1-\bar{u}_{i} q^{n+1}\right)}
$$

where $u$ is an $\operatorname{SO}(N, N)$ matrix with eigenvalues $\left\{u_{i}, \bar{u}_{i}\right\}_{i=1}^{N}$.

The next step of the argument consists of parametrising the different solutions for $l$ satisfying (3.28) in terms of $\mathrm{U}(N+1)$ and $\mathrm{U}(N)$ representations. Recall that the $\mathrm{U}(N)$ representations are labelled by pairs of Young diagrams $\boldsymbol{\Xi}=\left(\Xi_{l}, \Xi_{r}\right)$, where the corresponding $\mathrm{U}(1)$ charge is given by $|\boldsymbol{\Xi}|_{-}=\left|\Xi_{r}\right|-\left|\Xi_{l}\right|$. For a given $\mathrm{SU}(N)$ representation $\Xi$, there are different $\mathrm{U}(N)$ representations $\boldsymbol{\Xi}$ that restrict to $\Xi$; the $\mathrm{U}(1)$ charge of the various choices for $\boldsymbol{\Xi}$ differ by integer multiples of $N$. Since we may in particular take $\boldsymbol{\Xi}=(0, \boldsymbol{\Xi})$ and $\boldsymbol{\Lambda}=(0, \Lambda)$, it follows that a solution to $(3.28)$ is given by taking

$$
l=N|\boldsymbol{\Lambda}|_{-}-(N+1)|\boldsymbol{\Xi}|_{-} .
$$

The different possible solutions for $l$ are then accounted for by the different choices for lifting $\Lambda$ and $\Xi$ to $\mathrm{U}(N+1)$ and $\mathrm{U}(N)$ representations $\boldsymbol{\Lambda}$ and $\boldsymbol{\Xi}$, respectively, and thus (3.45) describes the most general solution. Actually, there is now a redundancy in our description since 'shifting' the separation between $\Lambda_{l}$ and $\Lambda_{r}$ in $\boldsymbol{\Lambda}$, and between $\Xi_{l}$ and $\Xi_{r}$ in $\boldsymbol{\Xi}$ by the same amount does not affect $l$. However, this redundancy disappears in the large $N$ limit, as there is then a unique way of identifying the two finite Young diagrams.

With this parametrisation in mind, we now define the $k$-independent function as

$$
s a_{\boldsymbol{\Lambda} ; \boldsymbol{\Xi}}^{N}=a_{\boldsymbol{\Lambda}_{N+1} ; \boldsymbol{\Xi}_{N}, N|\boldsymbol{\Lambda}|_{-}-(N+1)|\boldsymbol{\Xi}|_{-}}^{N},
$$

so that (3.43) becomes

$$
\operatorname{ch}_{\boldsymbol{\Lambda}}^{N+1}\left(\imath_{1}(v, w)\right) \vartheta\left(q, \imath_{2}(v, w)\right)=\sum_{\boldsymbol{\Xi}} s a_{\boldsymbol{\Lambda} ; \boldsymbol{\Xi}}^{N}(q) \operatorname{ch}_{\Xi}^{N}\left(v \bar{w}^{N+1}\right) .
$$

Note that for $\boldsymbol{\Lambda}=0$ this identity is just

$$
\begin{aligned}
\vartheta\left(q, \imath_{2}(v, w)\right) & =\prod_{n=1}^{\infty} \prod_{i=1}^{N} \frac{\left(1+v_{i} \bar{w}^{N+1} q^{n-\frac{1}{2}}\right)\left(1+\bar{v}_{i} w^{N+1} q^{n-\frac{1}{2}}\right)}{\left(1-v_{i} \bar{w}^{N+1} q^{n}\right)\left(1-\bar{v}_{i} w^{N+1} q^{n}\right)} \\
& =\sum_{\Xi} s a_{0 ; \Xi}^{N}(q) \operatorname{ch}_{\Xi}^{N}\left(v \bar{w}^{N+1}\right) .
\end{aligned}
$$


In order to describe the general case from this, let us introduce the restriction coefficients $r_{\boldsymbol{\Lambda} \Phi}^{(N)}$ as the multiplicities with which the $\mathrm{U}(N)$ characters appear in the decomposition of $\mathrm{U}(N+1)$ characters

$$
\operatorname{ch}_{\boldsymbol{\Lambda}}^{N+1}\left(\imath_{1}(v, w)\right)=\sum_{\boldsymbol{\Phi}} r_{\boldsymbol{\Lambda} \boldsymbol{\Phi}}^{(N)} \operatorname{ch}_{\mathbf{\Phi}}^{N}\left(v \bar{w}^{N+1}\right)
$$

as well as the $\mathrm{U}(N)$ Clebsch-Gordan coefficients

$$
\operatorname{ch}_{\boldsymbol{\Lambda}}^{N} \operatorname{ch}_{\Xi}^{N}=\sum_{\Pi} c_{\boldsymbol{\Lambda} \Xi}^{(N) \Pi} \operatorname{ch}_{\Pi}^{N}
$$

Then it follows from eq. (3.47) that we have

$$
s a_{\boldsymbol{\Lambda} ; \boldsymbol{\Xi}}^{N}(q)=\sum_{\boldsymbol{\Phi}, \boldsymbol{\Psi}} r_{\boldsymbol{\Lambda} \boldsymbol{\Phi}}^{(N)} c_{\boldsymbol{\Phi} \boldsymbol{\Psi}}^{(N) \Xi} s a_{0 ; \boldsymbol{\Psi}}^{N}(q)=\sum_{\boldsymbol{\Phi}, \boldsymbol{\Psi}} r_{\boldsymbol{\Lambda} \boldsymbol{\Phi}}^{(N)} c_{\boldsymbol{\Phi} \bar{\Xi}}^{(N) \bar{\Psi}} s a_{0 ; \boldsymbol{\Psi}}^{N}(q)
$$

Generalising the combinatorial calculation of section 2.4, we shall show in section 3.4 that the large $N$ limit of the branching functions equals

$$
\begin{aligned}
s a_{0 ; 0}(q) & =\lim _{N \rightarrow \infty} s a_{0 ; 0}^{N}(q)=\prod_{n=1}^{\infty} \frac{\left(1+q^{\frac{n}{2}+1}\right)^{2 n}}{\left(1-q^{n}\right)^{2 n-1}} \\
s a_{0 ; \Xi}(q) & =\lim _{N \rightarrow \infty} s a_{0 ; \Xi}^{N}(q)=s a_{0 ; 0}(q) \operatorname{sch}_{\Xi_{l}^{t}}\left(\mathcal{U}_{1}\right) \operatorname{sch}_{\Xi_{r}^{t}}\left(\mathcal{U}_{1}\right),
\end{aligned}
$$

where the $\mathrm{GL}(\infty \mid \infty)_{+}$supercharacters $\operatorname{sch}_{\Xi}$ were defined in (3.37) and $\mathcal{U}_{1}=\mathcal{U}\left(h=\frac{1}{2}\right)$. Notice that $\left|s a_{0 ; 0}(q)\right|^{2}=\mathcal{Z}_{\text {gauge }}(q)$.

In the final step of the argument we have to remove the null states that appear in the limit. By analogy with the bosonic case, we propose that this amounts to replacing the restriction and Clebsch-Gordan coefficients by

$$
\begin{gathered}
\lim _{N \rightarrow \infty} c_{\boldsymbol{\Phi} \bar{\Xi}}^{(N) \bar{\Psi}} \rightarrow c_{\Phi_{l} \Xi_{r}} c_{\Phi_{r}} \Xi_{l} \\
\lim _{N \rightarrow \infty} r_{\boldsymbol{\Lambda} \mathbf{\Phi}}^{(N)} \rightarrow r_{\Lambda_{l} \Phi_{l}} r_{\Lambda_{r} \Phi_{r}} .
\end{gathered}
$$

Here $c_{\Phi \Psi} \Xi$ are the Clebsch-Gordan coefficients of $\mathfrak{g l}(\infty)+$ that already appeared in section 2; as is explained in appendix A, we can also interpret them as $\mathfrak{g l}(\infty \mid \infty)_{+}$Clebsch-Gordan coefficients, i.e. they satisfy

$$
\operatorname{sch}_{\Lambda} \operatorname{sch}_{\Xi}=\sum_{\Pi \in Y} c_{\Lambda \Xi}^{\Pi} \operatorname{sch}_{\Pi}
$$

The coefficients appearing on the right hand side of eq. (3.56) are the restriction coefficients for $\mathfrak{g l}(\infty)_{+}$that can be expressed in terms of the Clebsch-Gordan coefficients as [62]

$$
r_{\Lambda \Xi}=c_{\Xi|\Lambda / \Xi|} \stackrel{\Lambda}{ }
$$

where $|\Lambda / \Xi|$ denotes the Young diagram with a single row of $|\Lambda|-|\Xi|$ boxes. In particular, eq. (3.58) implies that $r_{\Lambda \Xi}$ can only be either 0 or 1 . The coefficients $r_{\Lambda \Xi}$ also define 
restriction coefficients for $\mathfrak{g l}(\infty \mid \infty)_{+}$, since we have the identity (see appendix A for a detailed derivation)

$$
\operatorname{sch}_{\Lambda}\left(\mathcal{U}_{0}\right)=\sum_{\Xi \in Y} r_{\Lambda \Xi} \operatorname{sch}_{\Xi^{t}}\left(\mathcal{U}_{1}\right)
$$

were $\mathcal{U}_{0}=\mathcal{U}(h=0)$. Note that we are considering here the branching rules of $\mathfrak{g l}(\infty \mid \infty)_{+^{-}}$ representations into representations of the subalgebra of infinite matrices whose first row and column is zero; the latter algebra is again $\mathfrak{g l}(\infty \mid \infty)_{+}$, but with a shifted definition of parity, and this is the origin of the transposition of $\Xi$ on the right-hand-side.

With these preparations we can now finally compute the partition function for the Hilbert space

$$
\mathcal{H}_{s}^{\lambda}=\bigoplus_{\Lambda, \Xi \in \mathbf{Y}}(\boldsymbol{\Lambda} ; \boldsymbol{\Xi})_{s} \otimes{\overline{(\boldsymbol{\Lambda} ; \boldsymbol{\Xi})_{s}}}
$$

of the Kazama-Suzuki coset (3.16) in the 't Hooft limit (2.15), where we have denoted by $(\boldsymbol{\Lambda} ; \boldsymbol{\Xi})_{s}$ the large $N, k$ limit of the $\mathcal{W}_{N, k}$ representations

$$
(\boldsymbol{\Lambda} ; \boldsymbol{\Xi})_{s}=\lim _{N, k \rightarrow \infty}\left(\boldsymbol{\Lambda}_{N+1} ; \boldsymbol{\Xi}_{N},\left[N|\boldsymbol{\Lambda}|_{-}-(N+1)|\boldsymbol{\Xi}|_{-}\right]\right)
$$

using the same notation as in (3.46). Their characters can be computed from (3.42), and dropping the null-states as in (3.56) and (3.55) we obtain

$$
\begin{aligned}
\operatorname{Tr}_{(\boldsymbol{\Lambda} ; \Xi)_{s}} q^{L_{0}} & =q^{\frac{\lambda}{2}(|\boldsymbol{\Lambda}|-|\boldsymbol{\Xi}|)} \sum_{\boldsymbol{\Phi}, \boldsymbol{\Psi}} r_{\Lambda_{l} \Phi_{l}} r_{\Lambda_{r} \Phi_{r}} c_{\Phi_{l} \Xi_{r}}{ }^{\Psi_{r}} c_{\Phi_{r}} \Xi_{l} \Xi_{l} \\
& s a_{0 ; 0}(q) \operatorname{sch}_{\Psi_{l}^{t}}\left(\mathcal{U}_{1}\right) \operatorname{sch}_{\Psi_{r}^{t}}\left(\mathcal{U}_{1}\right) \\
& =q^{\frac{\lambda}{2}(|\boldsymbol{\Lambda}|-|\boldsymbol{\Xi}|)} \operatorname{sa}_{0 ; 0}(q) \operatorname{sch}_{\Lambda_{l}}\left(\mathcal{U}_{0}\right) \operatorname{sch}_{\Lambda_{r}}\left(\mathcal{U}_{0}\right) \operatorname{sch}_{\Xi_{l}^{t}}\left(\mathcal{U}_{1}\right) \operatorname{sch}_{\Xi_{r}^{t}}\left(\mathcal{U}_{1}\right) \\
& =s a_{0 ; 0}(q) \operatorname{sch}_{\Lambda_{l}}\left(\mathcal{U}_{+}\right) \operatorname{sch}_{\Lambda_{r}}\left(\mathcal{U}_{+}\right) \operatorname{sch}_{\Xi_{l}^{t}}\left(\mathcal{U}_{-}\right) \operatorname{sch}_{\Xi_{r}^{t}}\left(\mathcal{U}_{-}\right)
\end{aligned}
$$

where the $\mathrm{GL}(\infty \mid \infty)_{+}$matrices $\mathcal{U}_{ \pm}$have been defined in (3.36), and we have used that

$$
\lim _{N, k \rightarrow \infty}\left[h_{\Lambda}^{N+1, k}-h_{\Xi}^{N, k+1}-\frac{\left(N|\boldsymbol{\Lambda}|_{-}-(N+1)|\boldsymbol{\Xi}|_{-}\right)^{2}}{2 N(N+1)(N+k+1)}\right]=\frac{\lambda}{2}(|\boldsymbol{\Lambda}|-|\boldsymbol{\Xi}|) .
$$

Finally, summing over the different representations, we get

$$
\operatorname{Tr}_{\mathcal{H}_{s}^{\lambda}} q^{L_{0}} \bar{q}^{\bar{L}_{0}}=\mathcal{Z}_{\text {gauge }} \sum_{\boldsymbol{\Lambda}, \Xi}\left|\operatorname{sch}_{\Lambda_{l}}\left(\mathcal{U}_{+}\right) \operatorname{sch}_{\Lambda_{r}}\left(\mathcal{U}_{+}\right) \operatorname{sch}_{\Xi_{l}^{t}}\left(\mathcal{U}_{-}\right) \operatorname{sch}_{\Xi_{r}^{t}}\left(\mathcal{U}_{-}\right)\right|^{2}
$$

which reproduces indeed the partition function (3.38) of the higher spin theory.

\subsection{Free field realisation}

Thus we are left with proving the combinatorial identities (3.53) and (3.54); this can be done as in the bosonic case using free fields.

First we note that the right-hand-side of (3.48) equals the partition function of $N$ complex fermions and $N$ complex bosons, transforming in the fundamental and antifundamental representations of $\mathrm{U}(N)$. More specifically, let us denote by $\psi^{1}, \ldots, \psi^{N}$ and $\jmath^{1}, \ldots, \jmath^{N}$ the fermionic and bosonic modes in the fundamental representation of $\mathrm{U}(N)$, 
respectively; their complex conjugates, $\bar{\psi}^{1}, \ldots, \bar{\psi}^{N}$ and $\bar{\jmath}^{1}, \ldots, \bar{j}^{N}$ then transform in the anti-fundamental representation. The full Fock space is spanned by the states of the form

$$
\prod_{j=1}^{n_{\bar{\psi}}} \bar{\psi}_{-r_{j}-\frac{1}{2}}^{a_{j}} \prod_{k=1}^{n_{\psi}} \psi_{-s_{k}-\frac{1}{2}}^{b_{k}} \prod_{l=1}^{n_{\bar{j}}} \bar{\jmath}_{-t_{l}-1}^{c_{l}} \prod_{m=1}^{n_{j}} J_{-u_{m}-1}^{d_{m}} \Omega,
$$

where the mode numbers $r_{j}, s_{k}, t_{l}, u_{m}$ are non-negative integers. In order to determine (3.53) and (3.54) we need to count the multiplicities with which a specific $\mathrm{U}(N)$ representation appears in the Fock space.

We begin again by counting the states that transform in the trivial representation. By the fundamental theorem of classical invariant theory [58], these states are linear combinations of the 'basic' invariants

$$
\begin{aligned}
& \prod_{r, s=0}^{\infty}\left(\sum_{a=1}^{N} \bar{\psi}_{-r-\frac{1}{2}}^{a} \psi_{-s-\frac{1}{2}}^{a}\right)^{K_{r s}} \prod_{t, u=0}^{\infty}\left(\sum_{a=1}^{N} \bar{\jmath}_{-t-1}^{a} \jmath_{-u-1}^{a}\right)^{L_{t u}} \\
& \quad \times \prod_{t, s=0}^{\infty}\left(\sum_{a=1}^{N} \bar{\jmath}_{-t-1}^{a} \psi_{-s-\frac{1}{2}}^{a}\right)^{P_{t s}} \prod_{r, u=0}^{\infty}\left(\sum_{a=1}^{N} \bar{\psi}_{-r-\frac{1}{2}}^{a} \jmath_{-u-1}^{a}\right)^{Q_{r u}} \Omega
\end{aligned}
$$

where only finitely many multiplicities $K_{r s}, L_{t u}, P_{t s}, Q_{r u}$ are non-zero. Note that each $P_{t s}$ and $Q_{r u}$ can only be 0 or 1 because the fermionic invariants $\sum_{a} \bar{\jmath}^{a} \psi^{a}$ and $\sum_{a} \bar{\psi}^{a} \jmath^{a}$ square to zero. In the $N \rightarrow \infty$ limit all the states in (3.66) are linearly independent, and it is straightforward to count them, leading to eq. (3.53)

$$
\begin{aligned}
s a_{0 ; 0}(q) & =\prod_{r, s=0}^{\infty} \sum_{K=0}^{\infty} q^{(r+s+1) K} \prod_{t, u=0}^{\infty} \sum_{L=0}^{\infty} q^{(t+u+2) L} \prod_{t, s=0}^{\infty} \sum_{P=0}^{1} q^{\left(t+s+\frac{3}{2}\right) P} \prod_{r, u=0}^{\infty} \sum_{Q=0}^{1} q^{\left(r+u+\frac{3}{2}\right) Q} \\
& =\prod_{n=1}^{\infty} \frac{1}{1-q^{n}} \prod_{i, j=0}^{\infty} \frac{\left(1+q^{i+j+\frac{3}{2}}\right)^{2}}{\left(1-q^{i+j+2}\right)^{2}} \\
& =\prod_{n=1}^{\infty} \frac{1}{1-q^{n}} \prod_{s=2}^{\infty} \prod_{n=s}^{\infty} \frac{\left(1+q^{n-\frac{1}{2}}\right)^{2}}{\left(1-q^{n}\right)^{2}}=\prod_{n=1}^{\infty} \frac{\left(1+q^{n+\frac{1}{2}}\right)^{2 n}}{\left(1-q^{n}\right)^{2 n-1}}
\end{aligned}
$$

Finally, we need to count the multiplicity with which a specific $\boldsymbol{\Xi}$ representation of $\mathrm{U}(N)$ appears; again, the argument follows the same logic as in the bosonic calculation in section 2.4. Let us consider the subspace of states of the form (3.65) with a fixed number of modes $n_{\psi}, n_{\bar{\psi}}, n_{\jmath}, n_{\bar{\jmath}}$. Then a $\mathrm{U}(N)$ tensor of shape $\boldsymbol{\Xi}=\left(\Xi_{l}, \Xi_{r}\right)$ such that $\left|\Xi_{l}\right|=n_{\bar{\psi}}+n_{\bar{\jmath}}$ and $\left|\Xi_{r}\right|=n_{\psi}+n_{\text {J }}$ will appear with multiplicity 0 or 1 . The multiplicity will be precisely 1 if there is (i) a Young supertableau of shape $\Xi_{l}$ with bosonic or even entries from $\left\{2 t_{l}+2\right\}$, and fermionic or odd entries from $\left\{2 r_{j}+1\right\}$; and (ii) a Young supertableau of shape $\Xi_{r}$ with bosonic or even entries from $\left\{2 u_{m}+2\right\}$, and fermionic or odd entries from $\left\{2 s_{k}+1\right\} .{ }^{6}$ This is equivalent to the requirement that there are Young supertableaux of shape $\Xi_{l}^{t}$ and $\Xi_{r}^{t}$ with entries from $\left\{2 r_{j}\right\} \cup\left\{2 t_{l}+1\right\}$ and $\left\{2 s_{k}\right\} \cup\left\{2 u_{m}+1\right\}$, respectively. Summing over all possible mode numbers and different $n_{\psi}, n_{\bar{\psi}}, n_{\jmath}, n_{\bar{\jmath}}$ such that $n_{\psi}+n_{\jmath}=\left|\Xi_{r}\right|$ and

\footnotetext{
${ }^{6}$ The form of these entries is twice the conformal dimension of the modes in (3.65).
} 
$n_{\bar{\psi}}+n_{\bar{\jmath}}=\left|\Xi_{l}\right|$, their contribution to the branching function (3.54) can be written with the help of (3.37) in the compact form

$$
\operatorname{sch}_{\Xi_{l}^{t}}\left(\mathcal{U}_{1}\right) \operatorname{sch}_{\Xi_{r}^{t}}\left(\mathcal{U}_{1}\right)
$$

Multiplying these minimal states with all the invariant states (3.66), one generates all states transforming in $\boldsymbol{\Xi}$ in the Fock space. Thus the branching function (3.54) is indeed just the product of (3.69) with (3.68).

\section{Conclusions}

In this paper we have given strong evidence in favour of the supersymmetric higher spin duality that was proposed in [44]. In particular, we have shown that the 1-loop partition function of the supersymmetric higher spin theory on $\mathrm{AdS}_{3}$ can be reproduced from the 't Hooft limit of the dual $\mathcal{N}=2$ Kazama-Suzuki models. Our analysis follows in spirit closely [30], where the corresponding consistency check for the original bosonic duality of [29] was performed. The main technical advance is that we have managed to determine the branching functions (both in the bosonic as well as the supersymmetric case) from first principles, using a free field description. This point of view also sheds light on the origin of the underlying $\mathfrak{g l}(\infty)$ symmetry (resp. $\mathfrak{g l}(\infty \mid \infty)$ for the supersymmetric case) of the partition function.

In order to make sense of the limit theory (and to match with the AdS gravity answer) we have assumed by analogy with the bosonic case that certain CFT states become null and decouple in the 't Hooft limit. It would be interesting to check this directly (at least for the first few cases) by performing a similar analysis to what was done in [30]. In order to be able to perform this analysis, it will be important to understand the underlying symmetry algebra - i.e. the supersymmetric analogue of $W_{\infty}[\lambda]$, see [26] — in more detail. This would also allow for a more detailed test of the correspondence by comparing eigenvalues of the various higher spin zero modes. We hope to come back to these issues elsewhere.

\section{Acknowledgments}

The work of CC and MRG is supported in parts by the Swiss National Science Foundation. We thank Maximilian Kelm and Carl Vollenweider for useful discussions.

\section{A Identities for $\mathfrak{g l}(\infty \mid \infty)_{+}$}

In this appendix we want to prove (3.57) as well as (3.58) and (3.59).

Let $\operatorname{Sym}_{n}$ be the group of permutations of the integers $\{1,2, \ldots, n\}$. Its irreducible representations $S_{\Lambda}$ are indexed by partitions of $n$, that is Young diagrams $\Lambda$ with $|\Lambda|=n$. When restricted to the subgroup $\operatorname{Sym}_{m} \times \operatorname{Sym}_{n} \subset \operatorname{Sym}_{m+n}$, a representation $S_{\Lambda}$ of $\operatorname{Sym}_{m+n}$ decomposes as ([54], chapter 1$)$

$$
\operatorname{res} S_{\Lambda} \cong \bigoplus_{\Xi, \Pi} c_{\Xi \Pi}^{\Lambda} S_{\Xi} \otimes S_{\Pi}
$$


where the sum is over partitions $\Xi$ of $m$ and partitions $\Pi$ of $n$, and we used the symbol $\nabla$ to denote the tensor product between representations of different groups.

Let $V$ be the fundamental representation of $\mathrm{U}(M \mid N)$, and $V_{\Lambda}$ the irreducible $\mathrm{U}(M \mid N)$ covariant tensor of shape $\Lambda$. Clearly, the two groups $\operatorname{Sym}_{n}$ and $\mathrm{U}(M \mid N)$ act naturally on the tensor product $V^{\otimes n}$. Because the action of $\mathrm{Sym}_{n}$ commutes with the action of $\mathrm{U}(M \mid N)$, one can consider $V^{\otimes n}$ as a representation of the product group $\operatorname{Sym}_{n} \times \mathrm{U}(M \mid N)$. With respect to this latter action, one has the following decomposition into irreducible representations [60]

$$
V^{\otimes n} \cong \bigoplus_{\Lambda} S_{\Lambda} \otimes V_{\Lambda}
$$

where the sum runs over all partitions $\Lambda$ of $n$ that fit into a hook with arm width $M$ and leg width $N$ [61]. We shall call these partitions hook-shaped. This type of multiplicity free decomposition is known in the mathematical literature as a Schur-Weyl duality.

Consider now the decomposition of the representation $V^{\otimes m} \otimes V^{\otimes n}$ with respect to the product group $\operatorname{Sym}_{m} \times \operatorname{Sym}_{n} \times \mathrm{U}(M \mid N)$. Applying eq. (A.2), on the one hand, to the whole tensor product $V^{\otimes(m+n)}$ and, on the other, to each factor $V^{\otimes m}$ and $V^{\otimes n}$ separately, one arrives at

$$
\bigoplus_{\Lambda} \operatorname{res} S_{\Lambda} \otimes V_{\Lambda}=\bigoplus_{\Xi, \Pi} S_{\Xi} \otimes S_{\Pi} \otimes\left(V_{\Xi} \otimes V_{\Pi}\right)
$$

Decomposing the restricted representation into irreducibles as in eq. (A.1), we conclude that the tensor product of irreducible $\mathrm{U}(M \mid N)$ representations must be

$$
V_{\Xi} \otimes V_{\Pi} \cong \bigoplus_{\Lambda} c_{\Xi \Pi}^{\Lambda} V_{\Lambda}
$$

where all partitions are hook-shaped. Setting $M=N$ and taking $N \rightarrow \infty$ we arrive at eq. (3.57). Note that the restriction on the hook-shape disappears in this limit.

Finally, we want to prove (3.58) and (3.59), following MacDonalds's book [54] on symmetric functions. Let $X, Y \in \mathrm{GL}(\infty \mid \infty)_{+}$be two diagonal matrices, whose entries we label as

$$
\begin{aligned}
X_{2 i, 2 i} & =x_{i+1}, \quad X_{2 i+1,2 i+1}=\xi_{i+1} \\
Y_{2 i, 2 i} & =y_{i+1}, \quad Y_{2 i+1,2 i+1}=\eta_{i+1}
\end{aligned} \quad i \in \mathbb{N}_{0} .
$$

We define a Schur type symmetric function by

$$
s_{\Lambda}(x \mid \xi)=\operatorname{sch}_{\Lambda}(X)=\sum_{T \in \operatorname{STab}_{\Lambda}} \prod_{j \in T} X_{j j}(-1)^{j},
$$

where $x=\left(x_{1}, x_{2}, \ldots\right), \xi=\left(\xi_{1}, \xi_{2}, \ldots\right)$ are treated as formal indeterminate variables. Note that if we restricted the values of $x$ and $\xi$ by setting $x_{i}=0$ for $i>M$ and $\xi_{j}=0$ for $j>N$, then (A.6) becomes a $\mathrm{U}(M \mid N)$ character. From (3.34) and the definition of Young supertableaux in figure 4 it follows that

$$
s_{\Lambda}(x \mid \xi)=s_{\Lambda^{t}}(-\xi \mid-x) .
$$

Denoting $y=\left(y_{1}, y_{2}, \ldots\right)$ and $\eta=\left(\eta_{1}, \eta_{2}, \ldots\right)$, we can now rewrite (3.35) as

$$
\prod_{i, j} \frac{\left(1-x_{i} \eta_{j}\right)\left(1-y_{i} \xi_{j}\right)}{\left(1-x_{i} y_{j}\right)\left(1-\xi_{i} \eta_{j}\right)}=\sum_{\Lambda} s_{\Lambda}(x \mid \xi) s_{\Lambda}(y \mid \eta),
$$


where the left hand side is to be understood as a generating function. Next we repeat the argument of ([54], pg. 40-41). Let us introduce a third set of independent variables $z=\left(z_{1}, z_{2}, \ldots\right), \zeta=\left(\zeta_{1}, \zeta_{2}, \ldots\right)$, and consider the product which we can rewrite in two different ways as

$$
\begin{aligned}
\prod_{i, j} \frac{\left(1-z_{i} \xi_{j}\right)\left(1-z_{i} \eta_{j}\right)\left(1-\zeta_{i} x_{j}\right)\left(1-\zeta_{i} y_{j}\right)}{\left(1-z_{i} y_{j}\right)\left(1-\zeta_{i} \xi_{j}\right)\left(1-\zeta_{i} \eta_{j}\right)} & =\sum_{\Lambda} s_{\Lambda}(z \mid \zeta) s_{\Lambda}(x \cup y \mid \xi \cup \eta) \\
& =\sum_{\Xi, \Pi} s_{\Xi}(z \mid \zeta) s_{\Xi}(x \mid \xi) s_{\Pi}(z \mid \zeta) s_{\Pi}(y \mid \eta) \\
& =\sum_{\Lambda, \Xi, \Pi} s_{\Lambda}(z \mid \zeta)\left(c_{\Xi \Pi} s_{\Xi}(x \mid \xi) s_{\Pi}(y \mid \eta)\right)
\end{aligned}
$$

where we have used (3.57) in the last line; this leads to the important relation

$$
s_{\Lambda}(x \cup y \mid \xi \cup \eta)=\sum_{\Xi, \Pi} c_{\Xi \Pi}^{\Lambda} s_{\Xi}(x \mid \xi) s_{\Pi}(y \mid \eta)
$$

We now specialise to $y=(w, 0,0, \ldots)$ and $\eta=(0,0, \ldots)$. Then $s_{\Pi}(y \mid \eta)$ becomes a $\mathrm{U}(1)$ character, which is only non-zero provided that $\Pi$ has a single row, in which case it equals $w^{|\Pi|}$. Next, we choose $x$ and $\xi$ so that for all $i \in \mathbb{N}_{0}$

$$
\xi_{i+1}=\left(\mathcal{U}_{1}\right)_{2 i, 2 i}=q^{i+\frac{1}{2}}, \quad x_{i+1}=\left(\mathcal{U}_{1}\right)_{2 i+1,2 i+1}=-q^{i+1},
$$

where $\mathcal{U}_{1}$ is, as before, defined by $\mathcal{U}_{1}=\mathcal{U}\left(h=\frac{1}{2}\right)$ and we recall that $\mathcal{U}(h)_{j j}=(-1)^{j} q^{h+\frac{j}{2}}$, see eq. (3.36). Then the eigenvalues of $\mathcal{U}_{0}=\mathcal{U}(h=0)$ are

$$
\left(\mathcal{U}_{0}\right)_{00}=1, \quad\left(\mathcal{U}_{0}\right)_{2 i+2,2 i+2}=-x_{i+1}=q^{i+1}, \quad\left(\mathcal{U}_{0}\right)_{2 i+1,2 i+1}=-\xi_{i+1}=-q^{i+\frac{1}{2}},
$$

where again $i \in \mathbb{N}_{0}$. Setting $w=1$, it follows from (A.12), (A.7), (A.10) and (A.11) that

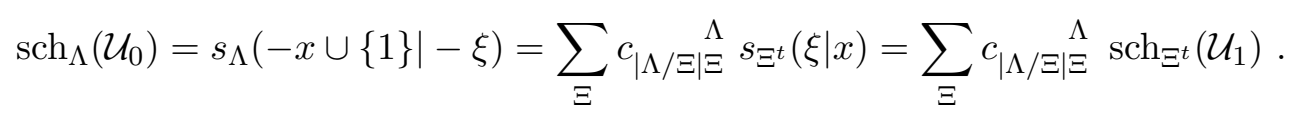

This completes the proof of (3.58) and (3.59).

\section{References}

[1] E. Fradkin and M.A. Vasiliev, Cubic interaction in extended theories of massless higher spin fields, Nucl. Phys. B 291 (1987) 141 [InSPIRE].

[2] E. Fradkin and M.A. Vasiliev, On the gravitational interaction of massless higher spin fields, Phys. Lett. B 189 (1987) 89 [INSPIRE].

[3] B. Sundborg, Stringy gravity, interacting tensionless strings and massless higher spins, Nucl. Phys. Proc. Suppl. 102 (2001) 113 [hep-th/0103247] [INSPIRE].

[4] E. Witten, talk at the John Schwarz 60-th birthday symposium, Pasadena U.S.A. (2001), http://theory.caltech.edu/jhs60/witten/1.html.

[5] A. Mikhailov, Notes on higher spin symmetries, hep-th/0201019 [INSPIRE]. 
[6] E. Sezgin and P. Sundell, Massless higher spins and holography, Nucl. Phys. B 644 (2002) 303 [Erratum ibid. B 660 (2003) 403] [hep-th/0205131] [INSPIRE].

[7] R. de Mello Koch, A. Jevicki, K. Jin and J.P. Rodrigues, $A d S_{4} / C F T_{3}$ construction from collective fields, Phys. Rev. D 83 (2011) 025006 [arXiv:1008.0633] [InSPIRE].

[8] M.R. Douglas, L. Mazzucato and S.S. Razamat, Holographic dual of free field theory, Phys. Rev. D 83 (2011) 071701 [arXiv:1011.4926] [INSPIRE].

[9] A. Jevicki, K. Jin and Q. Ye, Collective dipole model of AdS/CFT and higher spin gravity, J. Phys. A 44 (2011) 465402 [arXiv:1106.3983] [inSPIRE].

[10] A. Jevicki, K. Jin and Q. Ye, Bi-local model of AdS/CFT and higher spin gravity, arXiv:1112.2656 [INSPIRE].

[11] I. Klebanov and A. Polyakov, AdS dual of the critical $O(N)$ vector model, Phys. Lett. B 550 (2002) 213 [hep-th/0210114] [INSPIRE].

[12] E. Sezgin and P. Sundell, Holography in $4 D$ (super) higher spin theories and a test via cubic scalar couplings, JHEP 07 (2005) 044 [hep-th/0305040] [INSPIRE].

[13] M. Vasiliev, Nonlinear equations for symmetric massless higher spin fields in (A)dS(d), Phys. Lett. B 567 (2003) 139 [hep-th/0304049] [INSPIRE].

[14] M.A. Vasiliev, Higher spin gauge theories: star product and AdS space, in The many faces of the superworld, M.A. Shifman eds., World Scientific, Singapore (1999) [hep-th/9910096] [INSPIRE].

[15] X. Bekaert, S. Cnockaert, C. Iazeolla and M. Vasiliev, Nonlinear higher spin theories in various dimensions, hep-th/0503128 [INSPIRE].

[16] C. Iazeolla, On the algebraic structure of higher-spin field equations and new exact solutions, arXiv: 0807.0406 [INSPIRE].

[17] A. Campoleoni, Metric-like Lagrangian formulations for higher-spin fields of mixed symmetry, Riv. Nuovo Cim. 33 (2010) 123 [arXiv:0910.3155] [INSPIRE].

[18] S. Giombi and X. Yin, Higher spin gauge theory and holography: the three-point functions, JHEP 09 (2010) 115 [arXiv:0912.3462] [INSPIRE].

[19] S. Giombi and X. Yin, Higher spins in AdS and twistorial holography, JHEP 04 (2011) 086 [arXiv: 1004.3736] [INSPIRE].

[20] S. Giombi and X. Yin, On higher spin gauge theory and the critical $O(N)$ model, Phys. Rev. D 85 (2012) 086005 [arXiv: 1105.4011] [InSPIRE].

[21] J. Maldacena and A. Zhiboedov, Constraining conformal field theories with a higher spin symmetry, J. Phys. A 46 (2013) 214011 [arXiv:1112.1016] [INSPIRE].

[22] S. Prokushkin and M.A. Vasiliev, Higher spin gauge interactions for massive matter fields in 3 -D AdS space-time, Nucl. Phys. B 545 (1999) 385 [hep-th/9806236] [InSPIRE].

[23] S. Prokushkin and M.A. Vasiliev, 3-D higher spin gauge theories with matter, hep-th/9812242 [INSPIRE].

[24] M. Henneaux and S.-J. Rey, Nonlinear $W_{\text {infinity }}$ as asymptotic symmetry of three-dimensional higher spin Anti-de Sitter gravity, JHEP 12 (2010) 007 [arXiv: 1008.4579] [INSPIRE]. 
[25] A. Campoleoni, S. Fredenhagen, S. Pfenninger and S. Theisen, Asymptotic symmetries of three-dimensional gravity coupled to higher-spin fields, JHEP 11 (2010) 007 [arXiv: 1008.4744] [INSPIRE].

[26] M.R. Gaberdiel and T. Hartman, Symmetries of holographic minimal models, JHEP 05 (2011) 031 [arXiv:1101.2910] [INSPIRE].

[27] A. Campoleoni, S. Fredenhagen and S. Pfenninger, Asymptotic W-symmetries in three-dimensional higher-spin gauge theories, JHEP 09 (2011) 113 [arXiv:1107.0290] [INSPIRE].

[28] M.R. Gaberdiel, R. Gopakumar and A. Saha, Quantum W-symmetry in $A d S_{3}$, JHEP 02 (2011) 004 [arXiv: 1009.6087] [INSPIRE].

[29] M.R. Gaberdiel and R. Gopakumar, An AdS $S_{3}$ dual for minimal model CFTs, Phys. Rev. D 83 (2011) 066007 [arXiv: 1011.2986] [INSPIRE].

[30] M.R. Gaberdiel, R. Gopakumar, T. Hartman and S. Raju, Partition functions of holographic minimal models, JHEP 08 (2011) 077 [arXiv: 1106.1897] [INSPIRE].

[31] C.-M. Chang and X. Yin, Higher spin gravity with matter in $A d S_{3}$ and its CFT dual, JHEP 10 (2012) 024 [arXiv:1106.2580] [INSPIRE].

[32] K. Papadodimas and S. Raju, Correlation functions in holographic minimal models, Nucl. Phys. B 856 (2012) 607 [arXiv:1108.3077] [inSPIRE].

[33] C. Ahn, The coset spin-4 casimir operator and its three-point functions with scalars, JHEP 02 (2012) 027 [arXiv:1111.0091] [INSPIRE].

[34] A. Castro, R. Gopakumar, M. Gutperle and J. Raeymaekers, Conical defects in higher spin theories, JHEP 02 (2012) 096 [arXiv:1111.3381] [INSPIRE].

[35] M. Ammon, P. Kraus and E. Perlmutter, Scalar fields and three-point functions in $D=3$ higher spin gravity, JHEP 07 (2012) 113 [arXiv:1111.3926] [INSPIRE].

[36] C.-M. Chang and X. Yin, Correlators in $W_{N}$ minimal model revisited, JHEP 10 (2012) 050 [arXiv: 1112.5459] [INSPIRE].

[37] M.R. Gaberdiel and P. Suchanek, Limits of minimal models and continuous orbifolds, JHEP 03 (2012) 104 [arXiv:1112.1708] [INSPIRE].

[38] M. Gutperle and P. Kraus, Higher spin black holes, JHEP 05 (2011) 022 [arXiv:1103.4304] [INSPIRE].

[39] M. Ammon, M. Gutperle, P. Kraus and E. Perlmutter, Spacetime geometry in higher spin gravity, JHEP 10 (2011) 053 [arXiv: 1106.4788] [INSPIRE].

[40] P. Kraus and E. Perlmutter, Partition functions of higher spin black holes and their CFT duals, JHEP 11 (2011) 061 [arXiv:1108.2567] [INSPIRE].

[41] M.R. Gaberdiel, T. Hartman and K. Jin, Higher spin black holes from CFT, JHEP 04 (2012) 103 [arXiv: 1203.0015] [INSPIRE].

[42] C. Ahn, The large- $N$ 't Hooft limit of coset minimal models, JHEP 10 (2011) 125 [arXiv: 1106.0351] [INSPIRE].

[43] M.R. Gaberdiel and C. Vollenweider, Minimal model holography for $\mathrm{SO}(2 \mathrm{~N})$, JHEP 08 (2011) 104 [arXiv:1106.2634] [INSPIRE]. 
[44] T. Creutzig, Y. Hikida and P.B. Ronne, Higher spin AdS $S_{3}$ supergravity and its dual CFT, JHEP 02 (2012) 109 [arXiv:1111.2139] [INSPIRE].

[45] Y. Kazama and H. Suzuki, New $N=2$ superconformal field theories and superstring compactification, Nucl. Phys. B 321 (1989) 232.

[46] Y. Kazama and H. Suzuki, Characterization of $N=2$ superconformal models generated by coset space method, Phys. Lett. B 216 (1989) 112 [InSPIRE].

[47] S. Giombi, A. Maloney and X. Yin, One-loop partition functions of 3D gravity, JHEP 08 (2008) 007 [arXiv: 0804.1773] [INSPIRE].

[48] J.R. David, M.R. Gaberdiel and R. Gopakumar, The heat kernel on $A d S_{3}$ and its applications, JHEP 04 (2010) 125 [arXiv:0911.5085] [INSPIRE].

[49] P. Goddard, A. Kent and D. Olive, Virasoro algebras and coset space models, Phys. Lett. B 152 (1985) 88.

[50] P. Goddard, A. Kent and D.I. Olive, Unitary representations of the Virasoro and supervirasoro algebras, Commun. Math. Phys. 103 (1986) 105 [InSPIRE].

[51] D. Gepner, Field identification in coset conformal field theories, Phys. Lett. B 222 (1989) 207 [INSPIRE].

[52] W. Lerche, C. Vafa and N.P. Warner, Chiral rings in $N=2$ superconformal theories, Nucl. Phys. B 324 (1989) 427 [inSPIRE].

[53] G.W. Moore and N. Seiberg, Taming the conformal zoo, Phys. Lett. B 220 (1989) 422 [INSPIRE].

[54] I. MacDonald, Symmetric Functions and Hall Polynomials, Oxford University Press, Oxford U.K. (1979).

[55] P. Bouwknegt and K. Schoutens, $W$ symmetry in conformal field theory, Phys. Rept. 223 (1993) 183 [hep-th/9210010] [INSPIRE].

[56] D.J. Gross and W. Taylor, Two-dimensional QCD is a string theory, Nucl. Phys. B 400 (1993) 181 [hep-th/9301068] [INSPIRE].

[57] P. Di Francesco, P. Mathieu and D. Sénéchal, Conformal Field Theory, Springer, Berlin Germany (1997).

[58] H. Weyl, The Classical Groups: Their Invariants and Representations, Princeton University Press, Princeton U.S.A. (1939).

[59] K. Ito, Quantum Hamiltonian reduction and $N=2$ coset models, Phys. Lett. B 259 (1991) 73 [INSPIRE].

[60] A. Sergeev, Representations of the Lie-superalgebras gl $(n, m)$ and $Q(n)$ on the space of tensors, Funct. Anal. Appl. 18 (1984) 70 [inSPIRE].

[61] A. Berele and A. Regev, Hook Young-diagrams with applications to combinatorics and to representations of Lie-superalgebras, Adv. Math. 64 (1987) 118 [INSPIRE].

[62] R. King, Branching rules for classical Lie groups using tensor and spinor methods, J. Phys. A 8 (1975) 429. 